\title{
The near-infrared luminosity function of cluster galaxies beyond redshift one ${ }^{\star}$
}

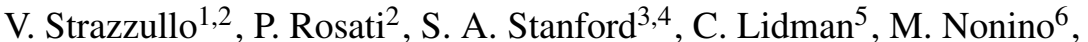 \\ R. Demarco ${ }^{7}$, P. E. Eisenhardt ${ }^{8}$, S. Ettori ${ }^{9}$, V. Mainieri ${ }^{10}$, and S. Toft ${ }^{11}$ \\ 1 Dipartimento di Scienze Fisiche, Università degli Studi di Napoli "Federico II", via Cinthia, 80126 Napoli, Italy \\ e-mail: vstrazzu@eso.org; strazzul@na.astro.it \\ 2 European Southern Observatory, Karl-Scwarzchild-Strasse 2, 85748 Garching, Germany \\ 3 Department of Physics, University of California at Davis, 1 Shields Avenue, Davis, CA 95616, USA \\ 4 Institute of Geophysics and Planetary Physics, LLNL, Livermore, CA 94551, USA \\ 5 European Southern Observatory, Alonso de Cordova 3107, Casilla 19001, Santiago, Chile \\ 6 Instituto Nazionale di Astrofisica, Osservatorio Astronomico di Trieste, via G.B. Tiepolo 11, 34131 Trieste, Italy \\ 7 Department of Physics and Astronomy, Johns Hopkins University, 3400 N. Charles Str., Baltimore, MD 21218, USA \\ 8 Jet Propulsion Laboratory, California Institute of Technology, MC 169-327, 4800 Oak Grove Drive, Pasadena, CA 91109, USA \\ 9 Istituto Nazionale di Astrofisica, Osservatorio Astronomico di Bologna, via Ranzani 1, 40127 Bologna, Italy \\ 10 Max-Planck-Institut für extraterrestrische Physik, Postfach 1319, 85748 Garching, Germany \\ 11 Department of Astronomy, Yale University, PO Box 208101, New Haven, CT 06520-8101, USA
}

Received 11 October 2005 / Accepted 16 January 2006

\section{ABSTRACT}

Aims. We determined the $K_{\mathrm{s}}$ band luminosity function (LF), and inferred the corresponding stellar mass function, of cluster galaxies at redshift $z \simeq 1.2$, using near-infrared images of three X-ray luminous clusters at $z=1.11,1.24,1.27$.

Methods. The composite LF was derived down to $M^{*}+4$, by means of statistical background subtraction, and is well described by a Schechter function with $K_{\mathrm{s}}^{*}=20.5_{-1}^{+0.4}$ and $\alpha=-1.0_{-0.3}^{+0.2}$. Using available X-ray mass profiles we determined the $M / L$ ratios of these three clusters, which tend to be lower than those measured in the local universe. Finally, from the $K_{\mathrm{s}}$ band composite LF we derived the stellar mass function of cluster galaxies.

Results. With these data, no significant difference can be seen between the cluster galaxies LF and the LF of field galaxies at similar redshift. We also found no significant evolution out to $z \simeq 1.2$ in the bright $\left(<M^{*}+4\right)$ part of the LF probed in this study, apart from a brightening of $\simeq 1.3$ mag of the characteristic magnitude of the high redshift LF. We confirm, and extend to higher redshift, the result from previous work that the redshift evolution of the characteristic magnitude $M^{*}$ is consistent with passive evolution of a stellar population formed at $z>2$.

Conclusions. The results obtained in this work support and extend previous findings that most of the stars in bright galaxies were formed at high redshift, and that $K_{\mathrm{s}}$-bright $\left(M>10^{11} M_{\odot}\right)$ galaxies were already in place at $z \simeq 1.2$, at least in the central regions of X-ray luminous clusters. Together with recent results on the field galaxy stellar mass function, this implies that most of the stellar mass is already assembled in massive galaxies by $z \simeq 1$, both in low and high density environments.

Key words. galaxies: clusters: general - Galaxy: evolution - Galaxy: formation - galaxies: luminosity function - mass function cosmology: observations

\section{Introduction}

Galaxy clusters are rare systems forming in the highest density peaks of large scale structure. In these special regions galaxy formation and evolutionary processes are expected to be faster

* Based in part on observations obtained at the European Southern Observatory using the ESO Very Large Telescope on Cerro Paranal (ESO program 166.A-0701). Based in part on observations obtained at the Hale Telescope, Palomar Observatory, as part of a continuing collaboration between the California Institute of Technology, NASA/JPL, and Cornell University. with respect to low density fields, thus making galaxy clusters a biased environment. On the other hand, clusters of galaxies, particularly at high redshift $(z \simeq 1)$, provide a convenient place for studying the evolution of massive galaxies. Not only do they contain high numbers of such objects, but these galaxies turn out to be so evolved (already at $z \simeq 1$ ) that they show a colourmagnitude sequence as clear as at lower redshifts. Thus evolved galaxies in distant clusters can be easily identified even without complete spectroscopic follow-up.

The study of massive galaxies has a relevant role in constraining galaxy formation and evolution models, as different 
models provide different predictions for their assembly (in particular in the redshift range $[0 \div 1])$. They could have rapidly formed their stars at high redshift and at the same time assembled their stellar mass, and then simply evolved passively as their stars aged. Alternatively, massive galaxies could have assembled on a longer time scale in a process of continuous merging of smaller units until redshift $<1$. Comparison of these different scenarios has proven to be a difficult task: even if merging galaxies are observed, the relevance of the merging process in galaxy evolution and especially the epoch at which major mergers occur is still debated.

Colours and spectra of massive galaxies at $z \simeq 1$ show that there is a significant population of such systems already hosting mainly evolved stellar populations, both in the field (see below) and in clusters (Stanford et al. 1997, 1998; van Dokkum et al. 1998; Benítez et al. 1999; De Propris et al. 1999; Rosati et al. 1999; Stanford et al. 2002; Blakeslee et al. 2003; van Dokkum \& Stanford 2003; Kodama et al. 2004; Lidman et al. 2004; De Lucia et al. 2004; Holden et al. 2005a,b; Tanaka et al. 2005). In such studies, the presence of evolved stellar populations is generally inferred from fundamental plane or colourmagnitude sequence evolution. These studies indicate that most of the stars in massive galaxies were formed at $z>2$. At the same time several published studies point out the less massive the galaxy is, the more likely is the presence of a younger component in its stellar population - the so called "downsizing" (Cowie et al. 1996) in galaxies hosting star formation.

It is not possible, however, on the basis of spectrophotometric analysis only, to rule out the possibility that these galaxies formed via merging of smaller galaxies with already evolved stellar populations even a short time before being observed. For instance, a passively evolving zero-point of the colourmagnitude relation does not imply that the galaxies formed long ago, but that the stars in the galaxies formed at high redshift, possibly in smaller progenitors.

In other words, while the underlying stellar populations can place constraints on the details of the star-formation history, they cannot tell when a galaxy assembled. In fact, even if these massive galaxies appear to be passively evolving, several studies have noted that to some extent they can still be forming (or recently have formed) stars (for instance Nakata et al. 2001; van Dokkum \& Stanford 2003; Holden et al. 2005c; Demarco et al. 2005; Jørgensen et al. 2005), likely implying merging (as observed for instance by van Dokkum et al. 1999 and Tran et al. 2005) or at least subsequent episodes of star formation. Such secondary episodes of star formation are probably correlated to cluster-related processes (accretion of field galaxies or groups and cluster merging), since galaxies which exhibit these features are often located outside of the cluster core, or in regions of lower X-ray luminosity. Moreover, the redshift evolution of the brightest cluster galaxies (BCGs) is peculiar and exhibits a large scatter at increasingly high redshift, so that at least in some cases merging could be required to make them evolve into local BCGs (see for instance Ellis \& Jones 2004). Even if it is difficult to trace a common "BCG evolutionary path", due to the intrinsically peculiar nature of these galaxies, some high redshift clusters (including Cl1252, Rosati 2004; Blakeslee et al. 2003; and Cl0848, van Dokkum et al. 2001) show signs of interactions or clear ongoing merging between massive galaxies which could lead to the formation of a cD.

However, even if the bulk of the stars had similar ages in the two formation scenarios (i.e. star formation occurring at the same early epoch), the epoch of assembly of the final mass observed locally in massive galaxies is different in the two models. If merging is a relevant process in the look-back time range that we can probe with observations, looking at progressively higher redshift should enable us to see the number of massive objects decreasing as they break up into their smaller progenitors, resulting in the evolution in the shape of the mass function of galaxies.

The differences in the predictions of the two formation scenarios have recently become less extreme, partly due to the higher redshift peak of the merging activity in $\Lambda$ CDM models as compared to standard CDM initially considered, but also due to ad hoc recipes for star formation adopted in the hierarchical merging models.

It has recently become more evident that, both in clusters (references mentioned above) and in the field (Glazebrook et al. 2004; McCarthy et al. 2004; Fontana et al. 2004; Saracco et al. 2004; Cimatti et al. 2002; Franx et al. 2003), a significant population of massive galaxies is already in place at $z \simeq 1 \div 2$. However, van Dokkum (2005) find that a considerable fraction of a nearby bulge-dominated galaxy sample recently experienced a merging episode involving more than $20 \%$ of its final mass. The stellar mass function of bright galaxies shows only a mild evolution up to redshift $\sim 1$, close to the prediction of simple pure luminosity evolution (e.g. Fontana et al. 2004).

The comparison with recent semi-analytical models however shows that different renditions predict very different evolution, especially at higher redshift (i.e. results are very sensitive to the chosen model ingredients), and most of them under-produce very massive galaxies (more severely the higher the redshift) even when reproducing the stellar mass function around $M_{*}$ - however, see also recent results from Bower et al. (2005). At the same time, Nagamine et al. (2005) show that with recent hydrodynamical simulations they can account for $\simeq 70 \%$ of the total stellar mass at $z=0$ already being formed by $z=1$.

Since a direct measure of the mass function is too difficult at high redshift for a reasonably large galaxy sample including faint objects, the near-infrared galaxy luminosity function (LF) represents an useful and cheaper surrogate. The galaxy LF is a first order description of a galaxy population (density of galaxies as a function of their luminosity). Despite (or because of) its conceptual simplicity the LF has been for many years one of the most popular tools for the interpretation of galaxy observations at all redshifts and in very different environments. The comparison of the LF at different redshifts constrains models of galaxy formation and evolution (Kauffmann \& Charlot 1998b), while the comparison of the LF in low and high density environments probes the relevance of the environmental effects on the galaxy populations. The LF is historically best studied in rich clusters of galaxies, which provide large numbers of galaxies at the same distance and, at low redshift, with high contrast against the background, allowing an efficient identification of cluster members with small contamination from background 
Table 1. Main properties of the cluster sample. Data are from Ettori et al. (2004).

\begin{tabular}{llllll}
\hline \hline Cluster & $z$ & $\begin{array}{l}T_{\text {gas }} \\
\mathrm{keV}\end{array}$ & $\begin{array}{l}R_{500} \\
\mathrm{kpc}\end{array}$ & $\begin{array}{l}L_{\text {bol }} \\
10^{44} \mathrm{erg} \mathrm{s}^{-1}\end{array}$ & $\begin{array}{l}M_{\text {tot }} \\
10^{14} M_{\odot}\end{array}$ \\
\hline RDCS J0910+5422 & 1.106 & $6.6_{-1.3}^{+1.7}$ & $818 \pm 150$ & $2.83 \pm 0.35$ & $4.91 \pm 2.93$ \\
RDCS J1252.9-2927 & 1.237 & $5.2_{-0.7}^{+0.7}$ & $532 \pm 40$ & $5.99 \pm 1.10$ & $1.59 \pm 0.35$ \\
RX J0848+4453 & 1.273 & $2.9_{-0.8}^{+0.8}$ & $499 \pm 115$ & $1.04 \pm 0.73$ & $1.37 \pm 0.98$ \\
\hline
\end{tabular}

Table 2. Summary of the principal characteristics of the NIR images used for the determination of the LF. Area $\mathrm{e}_{\mathrm{eff}}$ is the area of the region actually used in this work.

\begin{tabular}{|c|c|c|c|c|c|c|}
\hline Cluster & Telescope/Instrument & Filter & $\begin{array}{l}\text { Resolution } \\
\text { "! }\end{array}$ & $\begin{array}{l}\text { Completeness } \\
\text { AB mag }\end{array}$ & $\begin{array}{l}\text { Area }_{\mathrm{eff}} \\
\operatorname{arcmin}^{2} / \mathrm{Mpc}^{2}\end{array}$ & References \\
\hline RDCS J0910+5422 & Palomar $5 \mathrm{~m} / \mathrm{PFIC}$ & $\overline{K_{\mathrm{s}}}$ & 0.9 & 21.5 & $4.35 / 1.06$ & Stanford et al. (2002) \\
\hline RDCS J1252.9-2927 & VLT/ISAAC & $K_{\mathrm{s}}$ & 0.45 & 24.5 & $3.69 / 0.93$ & $\begin{array}{l}\text { Rosati et al. (2004) } \\
\text { Lidman et al. (2004) } \\
\text { Demarco et al. (in prep.) }\end{array}$ \\
\hline RX J0848+4453 & HST/NICMOS & F160W & $0 ! 22$ & 25 & $1.84 / 0.47$ & $\begin{array}{l}\text { Stanford et al. (1997) } \\
\text { Holden et al. (2004) } \\
\text { van Dokkum \& Stanford (2003) }\end{array}$ \\
\hline
\end{tabular}

galaxies. At higher redshift, the faint luminosities and the substantial background contamination makes the LF determination more uncertain. However, the steadily increasing data quality, and the quest for strict constraints on galaxy evolutionary models, have made the study of the LFs in high redshift clusters and fields a popular topic.

In this work, we determine the LF of distant $(z>1)$ cluster galaxies in the near-infrared (NIR). NIR galaxy samples are particularly well suited for studying galaxy evolution. Apart from advantages such as the smaller effect of dust extinction (as compared to bluer wavelengths), and the k-corrections relatively insensitive to galaxy type, they provide a relatively good estimate of the stellar mass in galaxies up to redshift $z \sim 2$. Therefore, near-infrared luminosity functions can trace the stellar mass function more effectively than bluer band LFs, which are more sensitive to the star formation histories of the galaxies.

While LFs for cluster galaxies at low redshift $(z \leq 0.2 \div 0.3)$ have been determined for a large number of clusters, allowing detailed discussion of the features and the separate contributions of different galaxy populations down to very faint magnitudes, the determination of the LF with comparable accuracy at high redshift is clearly more difficult.

The NIR LF of cluster galaxies at high redshift $(z \geq 0.8)$ has been measured by Trentham \& Mobasher (1998), De Propris et al. (1999), Nakata et al. (2001), Kodama \& Bower (2003), Toft et al. (2003), Ellis \& Jones (2004), and Toft et al. (2004). The evolution of the characteristic magnitude $M^{*}$ was first studied by De Propris et al. (1999) from low redshift up to $z \simeq 0.9$, finding that it is consistent with pure luminosity evolution of a stellar population formed at $z>2$; this result has been confirmed by subsequent studies. The evolution of the faint-end slope was only studied by Toft et al. $(2003,2004)$, who found a flatter slope at higher redshift compared to the local value.
The adopted cosmology in this paper is $H_{0}=$ $70 \mathrm{~km} \mathrm{~s}^{-1} \mathrm{Mpc}^{-1}, \Omega_{\mathrm{M}}=0.3, \Omega_{\Lambda}=0.7$ unless otherwise stated. Magnitudes are in the AB system.

\section{Data}

This work is mainly based on near-infrared images of three distant galaxy clusters: RDCS J1252.9-2927 at $z=1.24$ (hereafter Cl1252, Rosati et al. 2004), RX J0848+4453 at $z=1.27$ (hereafter C10848, Stanford et al. 1997), and RDCS J0910+5422 at $z=1.11$ (hereafter Cl0910, Stanford et al. 2002). The main properties of these three clusters are listed in Table 1. As the data used in this work have already been published, we refer the reader to the papers listed in Table 2 for details.

We used a $K_{\mathrm{s}}$ band image of the $\mathrm{Cl} 1252$ field obtained with the ISAAC infrared imager at the VLT (Lidman et al. 2004), a $K_{\mathrm{s}}$ band image of the $\mathrm{Cl0910}$ field obtained with the PrimeFocus Infrared Camera at the Palomar $5 \mathrm{~m}$ telescope (Stanford et al. 2002), and an $F 160 W$ ( $\simeq H$ band) image of the $\mathrm{Cl0848}$ field obtained with the NICMOS Camera 3 on the Hubble Space Telescope. While the quality of the two images for Cl1252 and Cl0848 is excellent (the PSF has FWHM $\simeq 0.45$ and 0.22 respectively, with limiting $\mathrm{AB}$ magnitude $\approx 25)$, the Cl0910 image has relatively poorer quality $(F W H M \simeq 0.9)$. While for both $\mathrm{Cl} 1252$ and $\mathrm{Cl0910}$ the field size of the portion of the image that we used has a radius $\simeq 65^{\prime \prime}$ (i.e. slightly more than $500 \mathrm{kpc}$ in linear scale), the NICMOS image for Cl0848 has a relatively small field (the maximum radius of the mosaic is $\simeq 55^{\prime \prime}$, i.e. $\simeq 450 \mathrm{kpc}$ at $z=1.27$ ). For all the images a catalog was produced with the SExtractor software (Bertin \& Arnouts 1996), and MAG_AUTO was used as a measure of the total magnitude. 

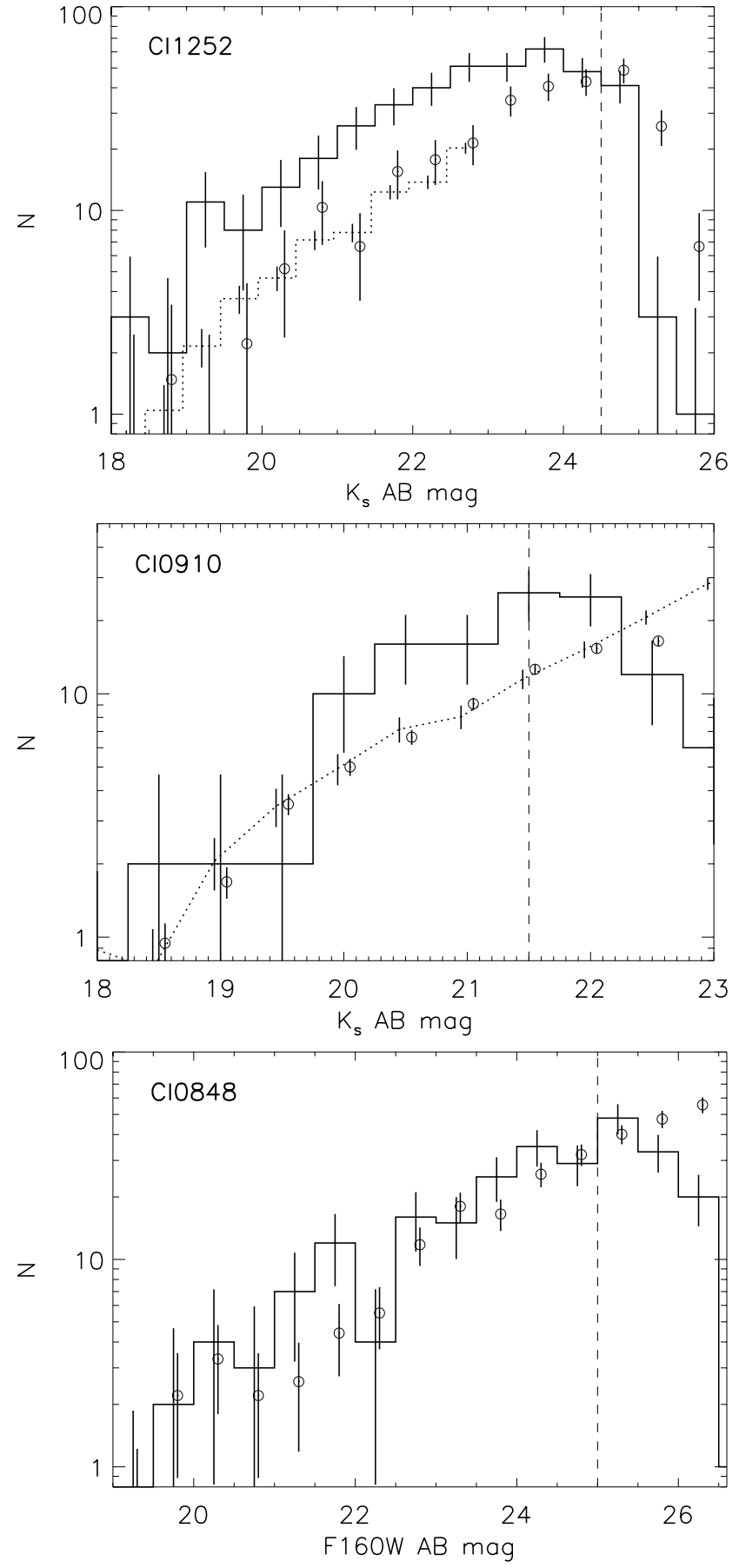

Fig. 1. Number counts of extended objects with $S / N>5$ in the clusters and reference fields. Upper panel: the solid line shows the number counts in the Cl1252 field ( $\simeq 3.7$ square arcmin). The circles and the dotted line show the counts in the reference regions selected in the FIRES and GOODS-S fields respectively, normalized to the cluster area. The counts in the GOODS-S field are shown down to the completeness magnitude $\left(K_{\mathrm{s}} \simeq 23\right)$. Middle panel: the solid line shows the number counts in the $\mathrm{C} 10910$ field $(\simeq 4.4$ square arcmin). The circles and the dotted line show the expected background level from reference regions selected in the SOFI and ISAAC GOODS-S fields, respectively. Lower panel: the solid line shows the number counts in the Cl0848 field ( $\simeq 1.8$ square arcmin), the circles show the expected background level based on the Hubble UDF. In all panels, the symbols/lines showing background number counts have been shifted by \pm 0.05 mag along the $x$-axis to avoid overlapping errorbars.
As discussed later, the galaxy luminosity function for all three clusters was determined by means of statistical subtraction of the fore- and background (hereafter background) contribution. Since the images are too small to estimate the local background from the images themselves, a control field was selected for each of the cluster fields in order to determine the background contribution to the galaxy counts. Ideally, the control field should be observed in the same filter and in very similar conditions and depths. For Cl1252 the control field is the FIRES (Faint Infrared Extragalactic Survey, Franx et al. 2000; Labbé et al. 2003) field in the HDF-S region, imaged with the same instrument and in the same filter as the cluster field. Because of its small area, we have complemented this field at bright magnitudes with a field in the GOODS-S region, also observed with VLT/ISAAC (Vandame et al., in preparation). For $\mathrm{Cl} 0848$ the reference field has been taken from the Hubble Ultra Deep Field (Thompson et al. 2005), also imaged with the same instrument and in the same filter as the C10848 field. For Cl0910 we had no control field available imaged with the same instrument, so we selected the reference field in the GOODS-S $K_{\mathrm{s}}$ images observed with both NTT/SOFI and VLT/ISAAC. Due to the similarity of the $K_{\mathrm{s}}$ band filters used for the GOODS and $\mathrm{Cl0910}$ images, we expect the background estimate to be appropriate. In particular, the SOFI image has comparable seeing ( $\simeq 0 . ' 9$ ) and comparable depth (see Fig. 1), and has the further advantage of being wider (thus smaller Poissonian errors).

For the purpose of identifying point-like sources we made use of the HST/ACS images available for all the cluster fields (Blakeslee et al. 2003; Postman et al. 2005) and for the reference regions in GOODS-S (Giavalisco et al. 2004) and HUDF (Beckwith et al., in preparation), and of the HST/WFPC2 for the reference region in the HDF-S field (Williams et al. 2000). In all catalogs, whenever possible the point-like sources were removed based on the MAG_AUTO vs. FLUX_RADIUS plot derived from the ACS images. The removed sources have $F W H M$ close to the PSF of the image. Point-like sources in regions not covered by the ACS image were identified in the SOFI image itself. Since point-like sources are a small fraction of the total counts, uncertainties in their removal have little effect on our results.

In order to estimate the luminosity function down to the faintest magnitude allowed by the data, the reference field has to be complete at least down to the completeness magnitude of the cluster field. In Fig. 1 we plot the number counts in the cluster and reference fields (normalized to the cluster field area). In all cases the completeness magnitude of the reference field is fainter or similar to that of the cluster field, therefore the following analysis is based on the cluster and reference fields catalogs down to the cluster field completeness magnitude, without completeness corrections. From the turn-over of the number counts for objects with $S / N$ ratio $>5$, the completeness magnitude of the cluster fields is estimated to be $K_{\mathrm{s}}=24.5$ for Cl1252, $K_{\mathrm{s}}=21.5$ for Cl0910, and $F 160 \mathrm{~W}=25$ for $\mathrm{Cl} 0848$.

This work also makes use of the extensive spectroscopic campaigns in these three clusters. We refer to Stanford et al. (1997, 2002), van Dokkum \& Stanford (2003) and Demarco et al. (in preparation) for details on the spectroscopic follow-up observations. 


\section{Luminosity functions}

For each of the clusters the luminosity function (LF) was calculated by means of statistical subtraction, i.e. using a reference field to remove the background contribution in the assumption that the field galaxy density is constant all over the sky. The statistical subtraction of the field galaxies is often considered to be an uncertain method of background removal especially at high redshifts, where the signal of the cluster against the background is progressively lower. However, while obtaining spectroscopic redshifts for all cluster galaxies down to a reasonably faint magnitude is clearly unfeasible, even the determination of photometric redshifts relies on the availability of deep photometry in several passbands, and on the assumption that the photometric redshift quality remains the same for spectral energy distributions (SED) for which no spectroscopic redshift can be measured.

Only one (Cl1252) of the three clusters studied has such a deep and wide photometric coverage (in addition to $38 \mathrm{spec}-$ troscopically confirmed members), that cluster membership determination fully based on photometric redshifts is feasible; the LF for Cl1252 was determined in this way by Toft et al. (2004, hereafter T04). Since for the other two clusters statistical subtraction is at present the only viable approach, the LF of Cl1252 was re-determined with this method, in the same region as in T04, as a first-order validation of the statistical subtraction procedure in this redshift range.

\subsection{LF determination}

For each cluster the LF was determined as follows. The galaxy counts in both the cluster and reference fields were binned, and the background contribution was estimated in each bin as the reference field counts normalized to the cluster area. For each bin, the error is estimated as the sum in quadrature of the Poissonian errors $1+(N+0.75)^{1 / 2}$ (Gehrels 1986) on both cluster and field counts in that bin.

The error on the background counts should also take into account the effects of galaxy clustering and of the lensing magnification of galaxies beyond the cluster. However, an estimate of the galaxy clustering contribution to the number counts error according to the prescriptions of Huang et al. (1997) yields a negligible difference with respect to the simple Poissonian error. Since a significant fraction of the observed galaxies are in the foreground of the cluster the effect due to lensing is likely small. Due to the large Poissonian errors, we can neglect these effects.

The existing spectroscopic data were taken into account, so that in each bin the background contribution contained at least as many galaxies as the spectroscopic interlopers, and the background corrected counts were at least equal to the number of confirmed members. This allows a more secure determination of the LF in the bright end, where due to low counts (both in the cluster and in the control fields) the statistical subtraction may be poor. In the area selected for the LF determination, most of the bright galaxies have measured redshifts: almost $80 \%$ of the galaxies down to $K_{\mathrm{s}}=21$ for $\mathrm{Cl} 1252$, more than $80 \%$ down to
$K_{\mathrm{s}}=21$ for $\mathrm{Cl0910}$, and more than $85 \%$ down to $F 160 \mathrm{~W}=22$ for $\mathrm{Cl0848.}$

Since the FIRES field is quite small, in order to achieve a better background evaluation (and smaller errors) in the bright end, the background estimate for C11252 from the FIRES field was supplemented with the estimate from the control field in GOODS-S (ISAAC) for magnitudes brighter than 21.5.

Due to the lower quality of the C10910 $K_{\mathrm{s}}$ image (seeing $\simeq 0$ ' 9 ), special care was taken for blended objects, particularly in the overdense cluster environment. Thanks to the availability of HST/ACS images in passbands F775W and F850LP, it was possible to crosscheck the catalogs to identify obvious blendings. Eight cases of evident blending were identified: for six of them, a more "aggressive" SExtractor configuration allowed the blended source to be split in sources located as in the ACS images. For the remaining 2 cases no deblending could be achieved, and as a zero-order approximation the flux from the source was split according to the flux ratio of the blended sources in the HST/ACS F850LP image.

The luminosity functions are shown in Fig. 2. The binned LFs were fit with the usual Schechter (1976) function with a maximum likelihood method using the Cash (1979) statistics $C=-2 \Sigma_{i}\left[n_{i} \ln \left(m_{i}\right)-m_{i}-\ln \left(n_{i} !\right)\right]$, where $n_{i}$ and $m_{i}$ are the observed number of galaxies in the $i$ th magnitude bin, and the number of galaxies predicted in the same bin by a Schechter function of parameters $M^{*}$ and $\alpha$, respectively. The best-fit $M^{*}$ and $\alpha$ are the parameters that minimize $C . \Phi^{*}$ was not taken as a free parameter, but was calculated for each choice of $M^{*}$ and $\alpha$ by requiring that the total number of predicted galaxies equal the number of those actually observed.

Even though the faint end slope cannot be well constrained (or is completely unconstrained, as in the case of C10910), due to the well known correlation of the Schechter parameters, leaving both $M^{*}$ and $\alpha$ free allows a better evaluation of the errors on $M^{*}$. The best-fit Schechter functions are overplotted on the LFs in Fig. 2. The best fitting Schechter parameters are listed in Table 3.

The maximum likelihood approach gives in principle an estimate of the confidence levels on the best-fit parameters. If the Cash statistic is defined as above, $\Delta C$ is distributed like $\Delta \chi^{2}$, thus $\Delta C=2.3,6.17$ gives the $1,2-\sigma$ confidence levels for two interesting parameters $M^{*}$ and $\alpha$. However, it should be noted that the Cash statistic should be applied to data which include background, because the background subtracted data are not Poisson-distributed, while the Cash statistic assumes Poisson probabilities. Even if we believe that the relevance of the $80 \%$ spectroscopic completeness on the LF bright end is important enough to adopt the previously described approach on binned, background subtracted counts, we note that in our LF fitting approach we hid the fact that the counts in each bin have errors larger than Poissonian due to the previous statistical background subtraction, and therefore we tend to underestimate the errors on $M^{*}$ and $\alpha$. For this reason, we also adopt a maximum likelihood approach on unbinned, not background subtracted data. Recently Andreon et al. (2005) summarized the principal reasons why one should adopt this approach, and proposed a method to be applied when the individual membership of the 

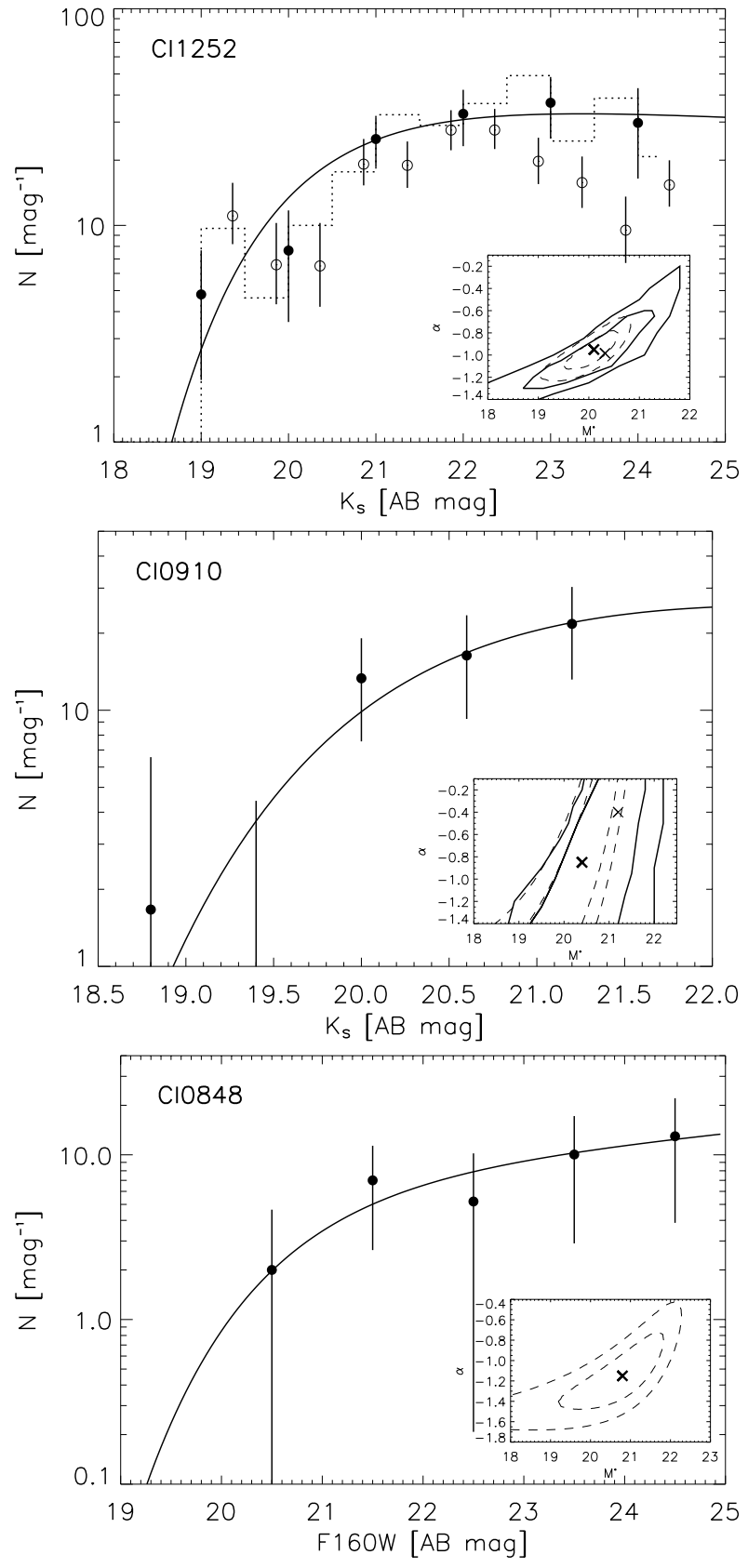

Fig. 2. Individual cluster luminosity functions for C11252 (upper panel), $\mathrm{Cl} 0910$ (middle), and Cl0848 (lower). In all panels, the solid line shows the best-fit Schechter function as derived from the binned counts, and the inserted plot shows the 1,2- $\sigma$ confidence levels on the parameters $M^{*}$ and $\alpha$, calculated from the maximum likelihood on binned counts (dashed lines) and from the maximum likelihood on unbinned, not background subtracted counts (solid thick lines - not shown for Cl0848, see text). The thick and thin crosses show the position of the best-fit parameters derived with the former and latter methods, respectively (see text for details). In the upper panel the empty circles show the Cl1252 LF as determined by T04, and the dotted histogram shows for comparison the LF from this work binned with the same bin size as in T04.

galaxies is unknown. When applying this method we are thus neglecting our redshift information, which means that we will derive conservative confidence intervals.
For each of the three clusters we applied the method described in Andreon et al. (2005), taking as a background dataset both the control regions in GOODS(ISAAC) and FIRES for C11252, and the control regions in GOODS(SOFI) and UDF for $\mathrm{Cl} 0910$ and $\mathrm{Cl} 0848$ respectively. In brief, we assume that the background number counts can be described by a power law (we use three parameters), and that the cluster LF is a Schechter function (also three parameters: $M^{*}, \alpha$, and $\Phi^{*}$ ). We then find the parameters that at the same time maximize the likelihood for the number counts in the control field (only described by the power law), and in the cluster field (described by the power law plus the Schechter function). In all cases, we first searched the complete 6 parameter space for the global maximum, and then found the maximum likelihood on a grid in the $M^{*}-\alpha$ plane (i.e. varying only the remaining four parameters), so that we can draw the confidence levels for these two parameters. Due to very low counts (as discussed below), it was difficult to maximize the likelihood against $M^{*}$ and $\alpha$ for C10848. Since the constraints on the Schechter parameters as determined with the first approach are already very loose, for this cluster we quote them in the following.

In Fig. 2 we show for reference the 1- and 2- $\sigma$ confidence levels obtained with the two different approaches for Cl1252 and C10910. As we mentioned, the smaller ones are understimated but the larger ones are likely overestimated, thus the "real" confidence levels are expected to lie between the two. The errors we quote in the following for Cl1252 and Cl0910 are derived from the larger ones.

The C11252 LF is shown together with the LF based on photometric redshifts from T04: the two determinations agree within the errors, with a larger discrepancy for the last magnitude bin. The difference between the two LFs could be considered a measure of the systematics of the two methods, with the error budget still dominated by low number statistics. The slope $\alpha$ determined via statistical subtraction $(-0.9 \pm 0.3)$ is somewhat steeper than the one determined via photometric redshifts $\left(\alpha=-0.64_{-0.25}^{+0.27}\right)$, however the two estimates are consistent within the errors. The $K_{\mathrm{s}}^{*}$ value $\left(20.1_{-1.2}^{+1.1}\right)$ is also found in agreement with the determination by T04 $\left(K_{\mathrm{s}}^{*}=20.41_{-0.55}^{+0.45}\right)$.

The C10910 Schechter function plotted in Fig. 2 is the formal best fit with $K_{\mathrm{s}}^{*}=20.4$ and $\alpha=-0.85$. As it is clear from Fig. 2, the slope $\alpha$ is unconstrained. Assuming $-1.4<\alpha<0.4$ yields an error of \pm 1 mag on $K_{\mathrm{s}}^{*}$.

Finally, some caveats apply to the determination of the Cl0848 LF. Namely, i) the very small field of view (less than 200 objects brighter than $H_{F 160 W} \simeq 25$ ), resulting in significant Poissonian errors, ii) the likely intrinsically lower richness of $\mathrm{Cl0848}$ compared to $\mathrm{Cl} 0910$ and $\mathrm{Cl1252}$ (since $\mathrm{Cl0848}$ has lower mass), iii) the presence of a known underlying supercluster in the Lynx field, and of a lower redshift cluster projected in the supercluster region (Stanford et al. 2001; Nakata et al. 2005). Mainly due to the first two reasons, the constraints on the Schechter parameters are quite loose, despite the significant depth of the $F 160 \mathrm{~W}$ image. The formal best-fit Schechter parameters are $F 160 W^{*}=20.8_{-1.6}^{+1 .}$ and $\alpha=-1.15_{-0.3}^{+0.4}$. 
Table 3. Luminosity function parameters and estimated absolute $K$ band luminosities and mass-to-light ratios. Column 2: indicative radius within which the LF and the $M / L$ ratio are measured; Col. 3: original passband in which the LF is measured; Col. 4: LF characteristic magnitude $M^{*}$ as measured in the original passband, Col. 5: $M^{*}$ k-corrected to the restframe $K_{\mathrm{s}}$ band ( $F 160 W$ was previously corrected to $K_{\mathrm{s}}$ band by a factor -0.5); Col. 6: LF faint end slope $\alpha$ (note that for C10910 $\alpha$ is unconstrained, and the error on $M^{*}$ for this cluster is estimated assuming that $-1.4<\alpha<-0.4$ ); Col. 7: the total restframe $K_{\mathrm{s}}$ luminosity within the effective area listed in Col. 6 of Table 2; Col. 8: the restframe $K_{\mathrm{s}}$ band mass-to-light ratio (the errors come from the errors on the projected mass and on the total luminosity). Note that for Cl1252 we report the total luminosity and the $M / L$ ratio derived from both the LFs from this work and from T04 (first and second value respectively).

\begin{tabular}{llllllll}
\hline \hline Cluster & $r$ & Passband & $M_{\mathrm{obs}}^{*}$ & $K_{\text {rest }}^{*}$ & $\alpha$ & $L_{K_{\mathrm{s}},<r}$ & $(M / L)_{K_{\mathrm{s}},<r}$ \\
& $\mathrm{kpc}$ & & $\mathrm{AB}$ mag & $\mathrm{AB} \mathrm{mag}$ & & $10^{12} L_{\odot}$ & $M_{\odot} / L_{\odot}$ \\
\hline RDCS J0910+5422 & 600 & $K_{\mathrm{s}}$ & $20.4_{-1.1}^{+1.2}$ & -23.27 & -0.85 & $10_{-1}^{+8}$ & $40_{-30}^{+20}$ \\
RDCS J1252.9-2927 & 500 & $K_{\mathrm{s}}$ & $20.1_{-1.2}^{+1.1}$ & -23.89 & $-0.95_{-0.35}^{+0.35}$ & $18_{-6}^{+5} \div 14_{-3}^{+3}$ & $13_{-5}^{+5} \div 16_{-5}^{+5}$ \\
RX J0848+4453 & 400 & $F 160 W(\simeq H)$ & $20.8_{-1.6}^{+1}$ & $-23.76_{-1.6}^{+1}$ & $-1.15_{-0.3}^{+0.4}$ & $4_{-1}^{+2}$ & $35_{-30}^{+25}$ \\
composite LF & - & $K_{\mathrm{s}}$ & $20.5_{-1}^{+0.4}$ & $-23.41_{-1}^{+0.4}$ & $-1.0_{-0.3}^{+0.2}$ & - & - \\
\hline
\end{tabular}

\subsubsection{Composite luminosity function}

Summing up the galaxy number counts of different clusters allows the background subtraction to be more effective (averaging over uncertainties in the statistical subtraction in each single cluster), and the shot noise to be reduced. The composite luminosity function was calculated in the observed $K_{\mathrm{s}}$ band at $z=1.2$. For this reason the $F 160 \mathrm{~W}$ magnitudes at $z=1.27$ were k-corrected to $K_{\mathrm{s}}$ magnitudes at $z=1.2$.

A single k-correction of 0.5 was applied at all magnitudes, as derived from synthetic SEDs (Bruzual \& Charlot 2003) of evolved simple stellar populations at that redshift $(3 \leq$ age $\leq$ 5 Gyr) No correction was made for the negligible k-correction between $z=1.2$ and $z=1.106$ or $z=1.237$. All the individual LFs were binned with a bin size of 0.5 mag (binning was adjusted to optimize individual magnitude coverage taking into account completeness limits and corrections for different redshift). $\mathrm{Cl} 1252$ and $\mathrm{Cl} 0848$ have the same " $K_{\mathrm{s}}$ " band completeness after these corrections, so their LFs were just summed up, and the errors were added in quadrature. Cl0910 instead is much shallower, so its LF was added to the composite LF up to its completeness magnitude. The composite LF beyond this magnitude is computed as the composite LF without Cl0910 multiplied by the ratio of the total counts (including all three clusters) to the total counts excluding C10910, computed in the magnitude interval where the $\mathrm{Cl} 0910$ photometry is complete (and errors were scaled accordingly). Due to the bright completeness limit for C10910, and the low counts for C10848, it is clear that the faint end of the composite LF is dominated by C11252. In our case, building the composite LF following other common methods as described in Colless (1989) or in Garilli et al. (1999), produces results consistent within 1- $\sigma$ with the LF calculated as above (note that the method described in Garilli et al. 1999, tends to give smaller errors, and a flatter slope than the one in Colless 1989).

The composite LF was also derived with a maximum likelihood approach on unbinned, not background subtracted data, as described in Andreon et al. (2005). However, since the LF of $\mathrm{Cl0848}$ is measured in a different passband than those of Cl1252 and $\mathrm{Cl} 0910, \mathrm{Cl} 0848$ was not included in this case. The composite LF was determined by fitting at the same time the counts in the two cluster fields (C11252 and C10910) and in all

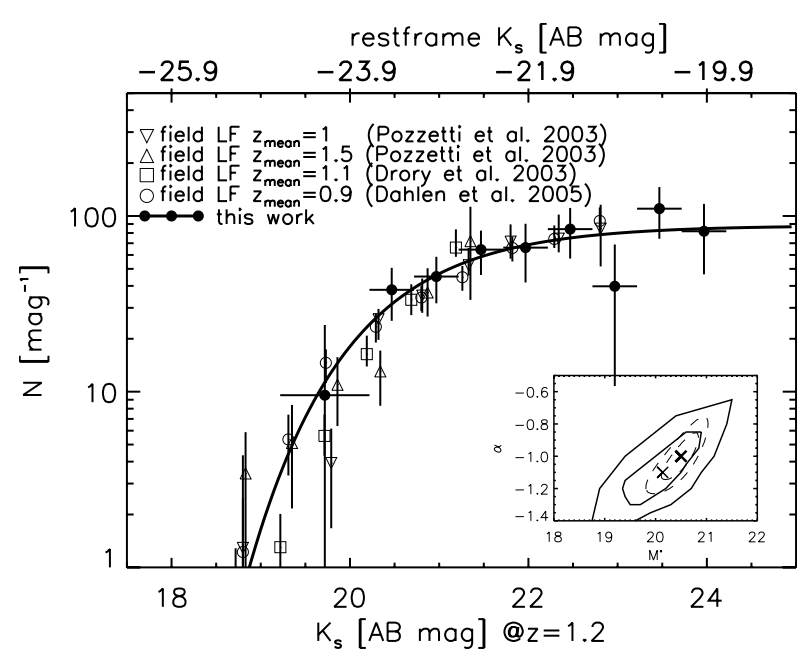

Fig. 3. The composite cluster luminosity function at $z=1.2$ (filled dots) compared to the field galaxies LF at $z \simeq 1$. The solid line shows the best-fit Schechter, and the inserted plot shows the 1,2- $\sigma$ confidence levels on the Schechter parameters as in Fig. 2. All field galaxies LFs have been arbitrarily rescaled.

the $K_{\mathrm{s}}$ band control fields. The two cluster LFs are assumed to be described by the same $M^{*}$ and $\alpha$ (but clearly have two different $\left.\Phi^{*}\right)$.

The composite luminosity function is shown in Figs. 3 and 4. The best-fit Schechter has $K_{\mathrm{s}}^{*}=20.5_{-1}^{+0.4}$ and $\alpha=$ $-1.0_{-0.3}^{+0.2}$. In Fig. 3 we also show the field LF at $z \simeq 1$ from Pozzetti et al. (2003), Drory et al. (2003) and Dahlen et al. (2005). In Fig. 4 the composite LF is compared to the local cluster galaxies $K$ band LF (corrected by 1.3 mag for passive evolution as derived below) as measured in Coma by De Propris et al. (1998) and Andreon \& Pelló (2000), and in samples of nearby clusters by Balogh et al. (2001) and Lin et al. (2004). All the local and field LFs have been arbitrarily rescaled. Both the Coma LFs shown were measured in the $H$ band and shifted to $K$ band with a colour term $H-K=0.24$. The $K$ band field LFs from Pozzetti et al. (2003) and Drory et al. (2003), and the $J$ band field LF from Dahlen et al. (2005), were converted to observed $K_{\mathrm{s}}$ magnitudes at $z=1.2$ by Eqs. (2) and (1) in Pozzetti et al. (2003). 


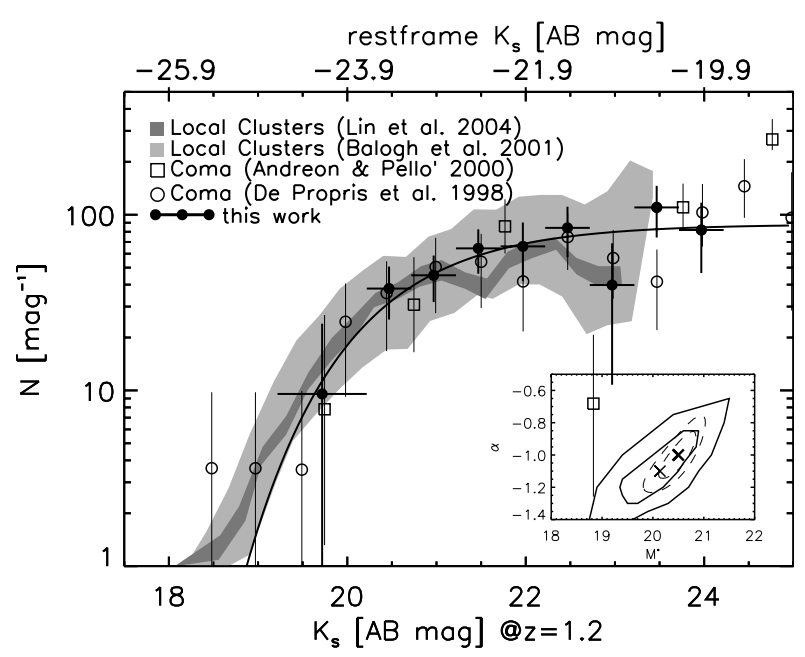

Fig. 4. The composite cluster luminosity function at $z=1.2$ (filled dots) compared to the local cluster galaxies LF, corrected by $1.3 \mathrm{mag}$ for passive evolution (see text). The solid line shows the best-fit Schechter, and the inserted plot shows the 1,2- $\sigma$ confidence levels on the Schechter parameters as in Fig. 2. All local cluster galaxies LFs have been arbitrarily rescaled.

With these data, no significant difference can be seen between the shapes of the cluster and field luminosity functions in the probed magnitude range, even if we find some evidence of an excess of very bright galaxies with respect to the field, as suggested for instance in De Propris et al. (2003).

The derivation of luminosity functions based on statistical subtraction may be affected by field-to-field variations, as the background evaluated from a control field may not be representative of the background in the cluster field. A robust estimate of such an effect requires adequately deep and large $K$ band fields. The VLT/ISAAC observations of the GOODS-S field, covering $\sim 100 \mathrm{arcmin}^{2}$, are currently the best data set available for this purpose. We note that part of this same field has been used as control field for determining the LFs of C10910 and Cl1252. In each of 17 selected ISAAC tiles in GOODS-S, we considered all galaxies within the central region (with the same area as the Cl1252 field used for the LF), down to the photometric completeness. We also considered in this case as an "additional tile" the FIRES HDF-S field we already used as control field for C11252.

In Fig. 5, we show the background number counts as estimated from different control fields: those from the FIRES field, which was used as the control field for C11252 (area $\simeq$ $5 \operatorname{arcmin}^{2}$ ) at faint magnitudes, those from the ISAAC GOODS-S mosaic used to complement the C11252 control field at bright magnitudes ( $\operatorname{area} \simeq 53 \mathrm{arcmin}^{2}$ ), those from the SOFI GOODS-S mosaic used for Cl0910 (area $\simeq 152 \mathrm{arcmin}^{2}$ ), and those from the 17 ISAAC tiles in GOODS-S (area $\simeq$ $3.7 \operatorname{arcmin}^{2}$ each).

We then built the $\mathrm{Cl} 0910$ and $\mathrm{C} 11252$ LFs as described above (making use of the spectroscopic information), based on these 18 small control fields, and for each of these we redetermined the composite LF. For Cl0848 we always used the LF determined above, since due to its very large uncertainties it has lower weight compared to $\mathrm{Cl0910}$ and C11252. Thus, we

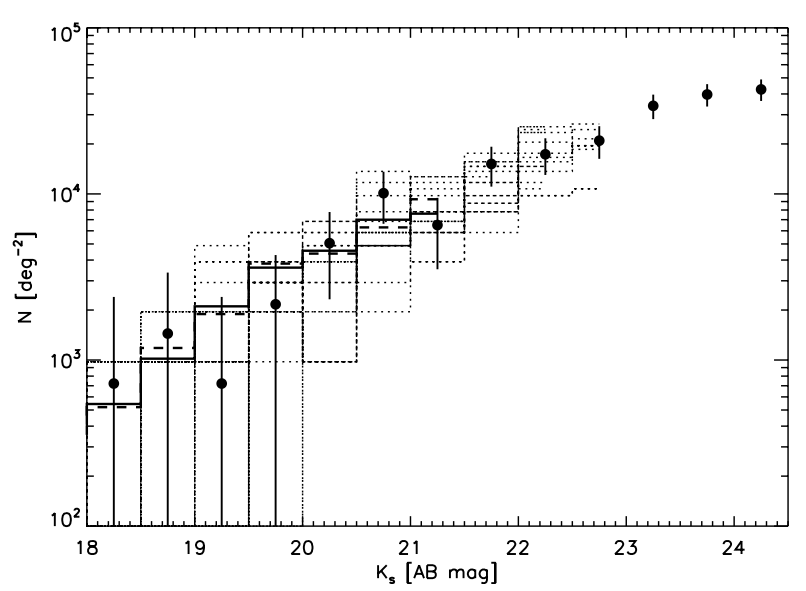

Fig. 5. The background number counts as estimated in different control fields. The filled symbols show the counts from the control field in the FIRES region, the solid and dashed lines from those in the GOODS-S regions (ISAAC and SOFI, respectively), and the dotted lines show the counts from the 17 ISAAC tiles in the GOODS-S field.

obtained 18 composite LFs corresponding to different control fields.

The GOODS-S ISAAC data, while being considerably wide, are not deep enough to reach our faint-end magnitudes. Therefore, we complemented the counts from each ISAAC tile at magnitudes fainter than its completeness with the FIRES counts. In this way, we probe field-to-field variations at magnitudes brighter than $K_{\mathrm{s}} \simeq 23$. The medians and standard deviations of $M^{*}$ and $\alpha$ obtained from these 18 determinations are $M^{*}=20.5 \mathrm{mag}, \sigma_{M^{*}}=0.14 \mathrm{mag}, \alpha=-1.0, \sigma_{\alpha}=0.06$.

In order to also account for field-to-field variations at fainter magnitudes (probed only by FIRES), we repeated the LFs determination by normalizing the FIRES counts at faint magnitudes by the ratio of the ISAAC/FIRES counts at magnitudes brighter than the completeness in each tile, assuming that the number density ratio is the same at fainter magnitudes. The medians and standard deviations of $M^{*}$ and $\alpha$ obtained from the 18 redeterminations with this procedure are $M^{*}=20.5 \mathrm{mag}$, $\sigma_{M^{*}}=0.09 \mathrm{mag}, \alpha=-1.0, \sigma_{\alpha}=0.09$.

In Fig. 6, we show the results from these two sets of tests. In the main panel, we show the confidence levels as shown in Figs. 3 and 4, and we overplot the Schechter parameters $M^{*}$ and $\alpha$ obtained for the 18 LFs. Since most of the data points overlap near the original determination marked by the cross, we show their distributions in the side-panels.

\subsection{Evolution of the restframe $K$ band $L F$}

It is customary to compare the observed evolution of $M^{*}$ with redshift with different predictions. Even if initially Barger et al. (1998) found no significant evolution for the infrared $M^{*}$ up to $z>0.5$, first De Propris et al. (1999) and then other works on high redshift clusters (Nakata et al. 2001; Kodama \& Bower 2003; Massarotti et al. 2003; Toft et al. 2003; Ellis \& Jones 2004) found that the evolution of $M^{*}$ up to $z \simeq 1$ is consistent with pure luminosity evolution of the cluster galaxies, and inconsistent with no-evolution predictions. As discussed in 


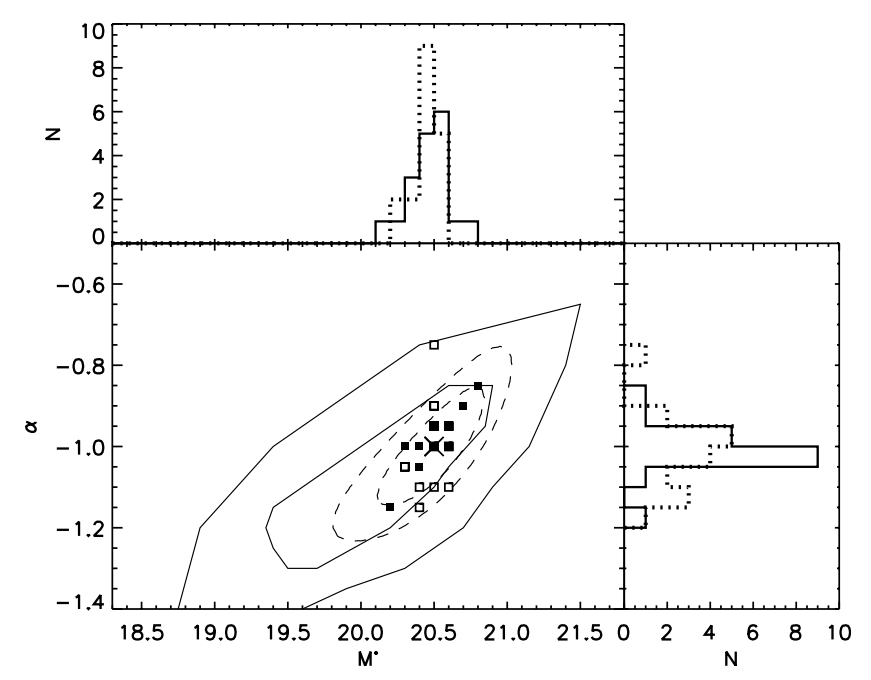

Fig. 6. Impact of field-to-field variations on the determination of the LF. In the main panel the best-fit $M^{*}$ and $\alpha$ (cross) and their 1- and 2- $\sigma$ confidence levels are shown, as in Figs. 3 and 4. The $M^{*}$ and $\alpha$ parameters from LF determinations with 18 different small control fields are shown as squares. In the side-panels the distributions of these $M^{*}$ (top) and $\alpha$ (right) are shown. Solid symbols (in the main panel) and solid lines (in the side panels) show the results for GOODS-S tiles counts complemented with FIRES counts at faint magnitudes, while empty symbols and dotted lines show the results with modified FIRES counts at faint magnitudes (see text for details).

Andreon (2001) and Andreon (2004), measuring an evolution in the LF from a change in the best-fit Schechter parameters is not straightforward. A density (clustercentric radius) dependent LF would imply a dependence of $M^{*}$ (and $\alpha$ ) on the surveyed area in different clusters at different redshifts, and the correlation of $M^{*}$ and $\alpha$ could introduce spurious results. This makes it difficult to study the LF evolution exclusively based on the evolution of the characteristic magnitude $M^{*}$. However, it is unlikely that the bright end of the LF is dominated by galaxies in the cluster outskirts, and even though the LF has indeed been found to be dependent on the sampled region within the cluster, this dependence mostly affects the LF at magnitudes fainter than those we can probe in this work (e.g. Popesso et al. 2005). When comparing LFs from different studies, we note that our $\mathrm{LF}$ is based on the central $r \leq 500 \mathrm{kpc}$ cluster regions, approximately corresponding to $r_{500}$, while the De Propris et al. (1998) $\mathrm{LF}$ is determined in the central $r \leq 350 \mathrm{kpc}$ Coma region, the Andreon \& Pelló (2000) LF is determined in a $\simeq 500 \times 500 \mathrm{kpc}^{2}$ region offset by $\simeq 360 \mathrm{kpc}$ from the Coma centre, and the Lin et al. (2004) LF is determined within the virial radius and is found to be very similar to the LF derived within $r_{500}$. Finally, if $\alpha$ is free in the Schechter fit, the errors on $M^{*}$ are reliable enough to make a fair comparison of the $M^{*}$ evolution with different predictions.

The comparison with previous determinations of $K_{\mathrm{s}}^{*}$ at lower and similar redshift is shown in Fig. 7. In agreement with previous work, the measured $K_{\mathrm{s}}^{*}$ is consistent with passive evolution predictions for an $L^{*}$ galaxy formed at $z \geq 2$. Converting the observed $K_{\mathrm{s}}^{*}$ to the absolute $K_{\mathrm{s}}$ band magnitude via $K_{\mathrm{s} \text {,rest }}=K_{\mathrm{s}, \text { obs }}-5 \log \left(d_{L} / 10 \mathrm{pc}\right)-\left(K_{\mathrm{s}, \text { rest }}-K_{\mathrm{s}, \mathrm{obs}}\right)_{z}$, as in

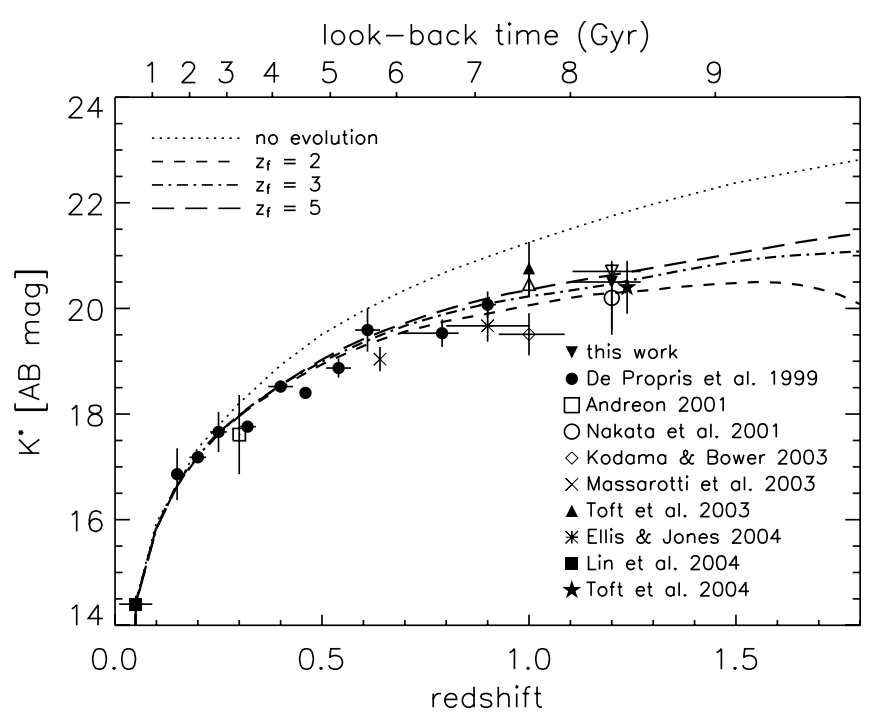

Fig. 7. The redshift evolution of the characteristic magnitude $K^{*}$. Different symbols show different determinations of $K^{*}$ as indicated in the legend. The no evolution prediction is calculated from the Coma $K^{*}$ (De Propris et al. 1998), k-corrected as in Pozzetti et al. (2003). Passive evolution model predictions are from Kodama \& Arimoto (1997), normalized to the Coma $K^{*}$. All determinations have fixed slope $\alpha=-0.9$, except Andreon (2001), Toft et al. (2003), Lin et al. (2004), T04 and the present work. For Lin et al. (2004) the plotted value is the mean of two values determined for $\alpha=-0.85$ and $\alpha=-1.1$. For both this work and Toft et al. (2003) also the $K^{*}$ value with $\alpha$ fixed at -0.9 is shown (corresponding empty symbol). Note that errors on $M^{*}$ which are computed with $\alpha$ fixed and free are not directly comparable. Error bars on the $x$-axis, when plotted, represent the redshift range of clusters which are combined to draw that point.

Pozzetti et al. (2003), gives $K_{\mathrm{s} \text {,rest }}^{*}=-23.41_{-1}^{+0.4}$. Compared to the Coma LF $K_{\mathrm{s}}^{*} \simeq-22.15$ (De Propris et al. 1998), this yields an evolution of $\Delta K^{*}=-1.3_{-1}^{+0.5}$.

As shown in Fig. 4, the shape of the composite LF is very similar to the shape of the local cluster galaxies LF shifted 1.3 mag brighter. This shape may be quantified in a nonparametric way by an analogue of the "giant-to-dwarf ratio" (GDR), which is defined in this case as the ratio of the number of galaxies brighter than $K_{\mathrm{s}}=21.2$ to the number of galaxies with $21.2<K_{\mathrm{s}}<24.2$. The $K_{\mathrm{S}}=21.2$ threshold corresponds to an absolute magnitude of $\simeq-22.7$, and to a stellar mass of $\simeq 8 \times 10^{10} M_{\odot}$ (for a Salpeter IMF). For our composite LF this GDR is $0.2 \pm 0.1$. If we estimate this GDR with the same absolute magnitude cut using the Coma LFs as shown in Fig. 4 (i.e. keeping into account the $1.3 \mathrm{mag}$ brightening), we similarly find a GDR of $0.2-0.3$. This suggests that a large fraction of the giant population was already in place at $z \sim 1$. We take note that a number of calculations including evolution corrections, k-corrections, and $H-K$ colour terms at redshift zero, are involved in such a comparison. We also note that we are applying a single value of 1.3 mag brightening for the whole LF, which is clearly a simplistic assumption, since galaxies with different star formation histories have different evolution corrections. 


\subsection{Contribution to the LF from early and late type galaxies}

The study of the early and late type galaxies LFs at this redshift is challenging.

Even when deep ACS data are available, (as in the case of C11252), morphological analysis is not feasible at the faint end $\left(z_{\mathrm{AB}} \gtrsim 25\right)$. Here we use the morphological information presented by Blakeslee et al. (2003), which is based on $i$ or $z$ band ACS imaging. Due to the red $B-z$ restframe colours (corresponding approximately to the observed $z-K$ ), it is not possible to build a morphological catalog down to the $K_{\mathrm{s}}$ band completeness magnitude. On the other hand, if early and late types are distinguished based on their colours, while the cluster red galaxies can still be isolated with relatively small background pollution, this does not hold for the blue population. For these reasons we only attempt a separation of the contributions to the LF from early and late type galaxies for the bright end of the C11252 LF.

For this cluster we can use the photometric members selected in T04, and distinguish early and late types based on the best-fitting template from photometric redshifts. The photometric redshifts in T04 were determined with 7 passbands against four templates (E/S0, Sbc, Scd, Irr) from Coleman et al. (1980), two starburst SEDs from Kinney et al. (1996), and interpolations between these six SEDs (see T04 for details). We then defined as early-types those galaxies best fitted with SEDs earlier than midway between $\mathrm{E} / \mathrm{S} 0$ and $\mathrm{Sbc}$ (i.e. roughly including E/S0 and possibly some Sa galaxies). We can then separate the two contributions to the LF, nearly down to the $K_{\mathrm{s}}$ band completeness magnitude, using the completeness function calculated by T04 (their Fig. 5) to correct both the early and late-types LFs for incompleteness due to photometric redshifts. While the reliability of photometric redshifts may be lower at the faint end, the early/late-type separation at the bright end is robust and the completeness correction negligible.

In Fig. 8 the early (filled dots) and late-types (empty circles) bright end LFs are shown, where the separation in early and late-types reflects their SED properties. We also show as a dashed line the LF of early type galaxies morphologically selected by Blakeslee et al. (2003) (brighter than $K_{\mathrm{s}}<22.5$, as the typical $z-K$ colours drive the sample beyond the completeness limit at fainter magnitudes). The morphologically and SED-selected early-types LFs are in very good agreement, suggesting that the bright end of the LF is already dominated by early-type galaxies, either selected on their morphology or on their spectrophotometric properties.

A histogram of the red-sequence galaxies (which are expected to be mostly early types) is also shown for comparison. This was derived taking all the galaxies (within $65^{\prime \prime}$ from the cluster center) with $i_{775}-z_{850}$ colours 0.16 mag redder or 0.14 mag bluer than the red sequence determined by Blakeslee et al. (2003). This is much larger than the intrinsic scatter found by Blakeslee et al. (2003), however the colours we used are 1". 5 aperture colours, so the scatter is expected to be larger. No statistical subtraction was attempted in this case, since a reference field with deep enough $K_{\mathrm{s}}, i_{775}$ and $z_{850}$ imaging is not available; however spectroscopic interlopers were removed. As

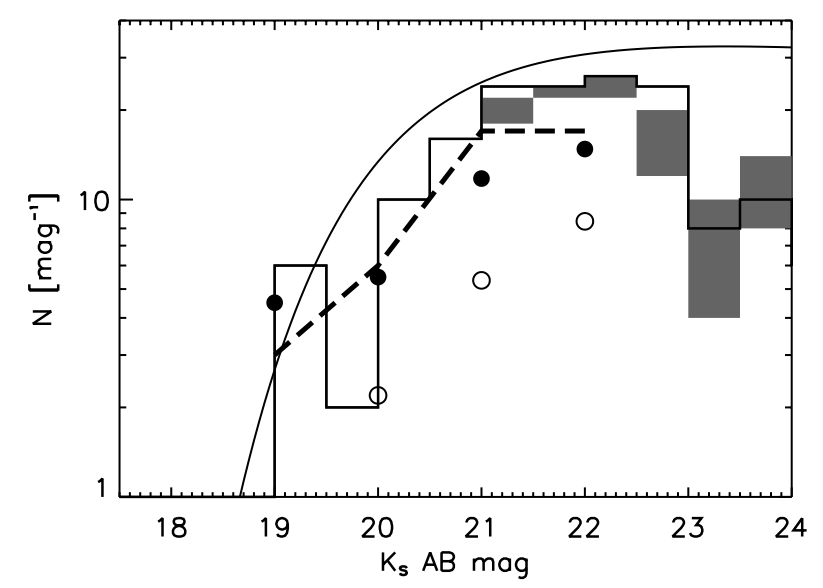

Fig. 8. The contribution of early and late type galaxies to the LF of C11252. The filled and empty symbols show the LFs for early and late type photometric members (based on the T04 sample), classified from their broad band colours. The dashed line shows the contribution of morphologically selected early-types (Blakeslee et al. 2003). The solid histogram shows the number counts of all galaxies (excluding spectroscopic interlopers) along the red sequence as determined in (Blakeslee et al. 2003) (no statistical subtraction of the field galaxies contamination was made; the shaded area shows the effect of photometric errors - see text). The solid curve shows the best-fit Schechter function for the Cl1252 global LF.

a result, the histogram shown is an upper limit to the effective LF of red sequence galaxies. The shaded area represents the 16-84 percentile variations of this histogram due to photometric errors, and was derived by simulating 100 catalogs where the $i_{775}-z_{850}$ colour was randomly shifted within a Gaussian of $\sigma$ equal to the photometric error on the $i_{775}-z_{850}$ colour. This histogram also confirms that the LF bright end is largely dominated by galaxies hosting evolved stellar populations.

A solid determination of the LF of red-sequence galaxies would require even more extensive redshift information at the faint end, which is however beyond the current spectroscopic limit. With our data, we observe some evidence of a deficit of faint galaxies on the red-sequence, which has been reported in other studies (Kajisawa et al. 2000; Nakata et al. 2001; Kodama et al. 2004; De Lucia et al. 2004; Tanaka et al. 2005), and is usually interpreted as a sign of downsizing.

\subsubsection{Contribution from clusters to the bright galaxies budget}

A rough estimate of the contribution of the bright cluster galaxies $\left(<M^{*}+2\right)$ to the total bright $\left(<M^{*}+2\right)$ galaxy budget can be obtained, by combining the cluster galaxies LF (and its measured evolution) with the known space density of clusters out to $z \sim 1$.

Our results show that at least at magnitudes brighter than $M^{*}+2$, the LF of the cluster galaxies appears to evolve mainly by passive evolution up to $z \simeq 1$. In addition, the normalization of the $K$ band galaxy LF of X-ray luminous clusters was found to be consistent with the local one at least out to $z \simeq$ 0.8 (Trentham \& Mobasher 1998). Therefore, we can assume that high-redshift clusters of a given X-ray luminosity contain 
similar numbers of bright $\left(<M^{*}+2\right)$ galaxies as low-redshift clusters of the same X-ray luminosity.

We use the relation between the number of galaxies brighter than $M^{*}+2$ within $r_{200}$, and the cluster mass within $r_{200}$, as derived by Popesso et al. (in preparation):

$N_{\text {galaxies }} \simeq 10^{-7.12}\left(M_{200} / M_{\odot}\right)^{0.61}$

and the relation between the X-ray luminosity and the cluster mass within $r_{200}$ (Reiprich \& Böhringer 2002), in the form:

$\log \left[\frac{L_{\mathrm{X}(0.1-2.4 \mathrm{keV})}}{h_{50}^{-2} 10^{40} \mathrm{erg} \mathrm{s}^{-1}}\right] \simeq-19+1.58 \log \left(\frac{M_{200}}{h_{50}^{-1} M_{\odot}}\right)$.

By combining these two scaling relations (and converting to $H_{0}=70 \mathrm{~km} \mathrm{~s}^{-1} \mathrm{Mpc}^{-1}$, and $\left.L_{\mathrm{X}}(0.5-2.0 \mathrm{keV})\left[10^{44} \mathrm{erg} \mathrm{s}^{-1}\right]\right)$, one obtains the number of bright galaxies in a cluster of luminosity $L_{X}$ :

$N\left(L_{X}\right) \simeq 74.5 \cdot\left(L_{X}(0.5-2.0 \mathrm{keV})\left[10^{44} \mathrm{erg} \mathrm{s}^{-1}\right]\right)^{0.386}$.

In the assumption that this holds in the redshift range $[0 \div 1]$, we can use the redshift evolution of the cluster X-ray luminosity function (Rosati et al. 2002; Mullis et al. 2004) to estimate the number density of clusters at a given luminosity and a given redshift. By using the $N\left(L_{\mathrm{X}}\right)$ relation above, we can compute the number density of bright galaxies in the cluster virial regions at each redshift. A comparison with the field LF (we used Trentham et al. 2005, at $z \simeq 0$, and Dahlen et al. 2005, at $z \simeq 1$ ) yields the contribution of cluster galaxies to the total bright galaxy budget. We thus find that a fraction of less than $10 \%(\simeq 6 \div 7 \%)$ of the bright galaxies at $z \simeq 0$ is located in the virial regions of X-ray luminous clusters (in broad agreement with what reported in De Propris et al. 2003), and similar $(\simeq 5 \%)$ at $z \simeq 1$.

\section{Mass to light ratios}

The cluster dynamical mass-to-light $(M / L)$ ratio has long been a matter of interest due to the fact that clusters collapse from regions several Mpcs wide, thus carrying both their mass and galaxy content from representative portions of the Universe. Their $M / L$ ratio could thus be similar to that of the whole Universe, even though this is strongly dependent on how different galaxy evolution is in such high density environments with respect to average density regions.

The cluster $M / L$ ratio can in principle be used to study how galaxies evolve in dense environments. The $M / L$ ratio is known to increase with the system mass going from galaxies to clusters (Bahcall et al. 1995), in agreement with the predictions of models of biased galaxy formation (Davis et al. 1985; Bardeen et al. 1986). However, it is not yet completely clear, also in recent studies, whether a mass dependence of the $M / L$ ratio is observed in the limited mass range of groups and clusters. While some works find a measurable increase of the $M / L$ ratio with the total mass of the system (for instance Schaeffer et al. 1993; Adami et al. 1998b,a; Girardi et al. 2000; Hoekstra et al. 2001; Girardi et al. 2002; Marinoni \& Hudson 2002; Bahcall \& Comerford 2002; Lin et al. 2003, 2004; Rines et al. 2004;
Tully 2005), some others conclude that the $M / L$ ratio is approximately the same in groups and clusters (for instance Dressler 1978; David et al. 1995; Carlberg et al. 1996, 1997; Cirimele et al. 1997; Hradecky et al. 2000).

As observed for instance in Tully (2005), galaxy groups with lower $B$ band $M / L$ ratios compared to more massive systems generally have a population of late type galaxies with on-going star formation. Moreover, systems with predominant E/S0/Sa population tend to have larger $M / L$ ratios; this would be consistent with more dense regions forming earlier, and therefore ending their star formation earlier. More recently, the $K$ band $M / L$ ratio, having very little dependence on on-going star formation, has been found to be mass-dependent (Lin et al. 2003, 2004), with $K$ band light per unit mass being higher by a factor $\simeq 2$ in low mass clusters than in massive ones.

As discussed for instance in Rines et al. (2004), the observed mass dependence could be due either to processes like tidal stripping and dynamical friction disrupting galaxies in massive clusters, or to a reduced star formation efficiency in such systems (possibly the heating of the ICM cutting off the supply of cold material needed to form stars, Blanton et al. 1999; Balogh et al. 2000).

Keeping in mind these issues that complicate the comparison of inhomogeneous samples, as well as possible systematic differences in the masses estimated by different means (see for instance Sanderson \& Ponman 2003; Andernach et al. 2004), we can compare the $M / L$ ratio of the clusters studied in this work with estimates at lower redshift. A comparison of the cluster $M / L$ ratios in the $B$ band, out to redshift $z \simeq 0.8$, was presented for instance by Hoekstra et al. (2002), who found that the evolution of the $M / L$ ratio is consistent with the luminosity evolution of galaxies as derived from the fundamental plane in distant clusters (see their Fig. 14).

We have estimated the $K$ band $M / L$ ratios of Cl0910, C11252, Cl0848 making use of the LFs derived above and of the X-ray mass profiles derived in Ettori et al. (2004).

Once the Schechter parameters $\alpha$ and $K_{\text {rest }}^{*}$ (and the corresponding characteristic luminosity $L^{*}$ ) have been determined, the $K$ band projected total luminosity within the surveyed area can be calculated via direct integration of the Schechter function $\left(L_{\text {tot }}=\Phi^{*} L^{*} \Gamma(2+\alpha)\right)$. The $K$ band luminosities for the three clusters within the surveyed area (as listed in Col. 6 of Table 2) are listed in Table 3. We assume $K_{\odot}=5.2$ when calculating luminosities in units of $L_{\odot}$. The errors were determined by calculating the luminosity with Schechter parameters running on the 1- $\sigma$ confidence level for $K^{*}$ and $\alpha$.

The projected $M / L$ ratios for the three clusters were derived within the surveyed area, using the projected mass profiles derived in Ettori et al. (2004, see Table 3).

Note that the mass to light ratio derived for $\mathrm{Cl} 1252$ by summing up the luminosities of the photometric members from T04 without further corrections $\left(M / L_{K}=17_{-3}^{+3} M_{\odot} / L_{\odot}\right)$ is consistent with the quoted value. By considering only the confirmed spectroscopic members, we obtain $M / L_{K}=31 \pm 5 M_{\odot} / L_{\odot}$, which should be considered as an upper limit.

It should be noted that, since our surveyed areas are different from each other and are small, we rely on the assumption of a negligible or small dependence of the $M / L$ ratio on 


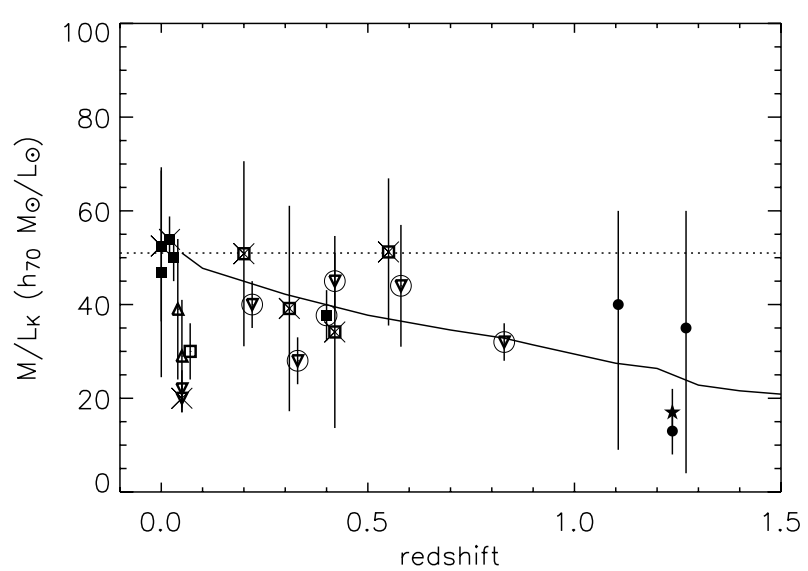

Fig. 9. The evolution of the restframe $K$ band mass-to-light ratio. The filled circles are the $M / L$ ratios from this work. The star shows the $M / L$ determined for $\mathrm{Cl1252}$ using the T04 LF. The filled squares are $K$ band $M / L$ measurement from Rines et al. (2001), Kneib et al. (2003), Lin et al. (2003), Rines et al. (2004). The empty symbols show the $K$ band $M / L$ ratio derived from published $M / L$ estimates in other passbands, i.e. $B / B_{j}$ band (circle) from Girardi et al. (2000), Hoekstra et al. (2002), Sanderson \& Ponman (2003), Gavazzi et al. (2004), $R / r$ band (square) from Carlberg et al. (1997) and Andernach et al. (2004), and $V$ band from Cirimele et al. (1997) and Hradecky et al. (2000) (see text). Crossed and circled points have masses determined from galaxy kinematics or strong/weak lensing respectively, at a difference with $\mathrm{X}$-ray masses used in this work. The solid line traces the evolution expected from pure luminosity evolution of a simple stellar population formed at $z=5$, assuming that the cluster $M / L$ ratio only evolves because of stellar evolution in galaxies, and neglecting any evolution in the dark mass. The dotted line shows the reference $(z=0)$ value.

the clustercentric distance within the virial radius (Rines et al. 2001; Kneib et al. 2003; Rines et al. 2004), when comparing the $M / L$ ratios derived here with other measurements.

In Fig. 9 the $K$ band $M / L$ ratio for the three clusters is compared to previous determinations at lower redshifts (Carlberg et al. 1997; Cirimele et al. 1997; Girardi et al. 2000; Hradecky et al. 2000; Rines et al. 2001; Hoekstra et al. 2002; Kneib et al. 2003; Lin et al. 2003; Sanderson \& Ponman 2003; Andernach et al. 2004; Gavazzi et al. 2004; Rines et al. 2004).

The $M / L$ ratios published in passbands different from $K$ were rescaled to the $K$ band using the colours of a simple stellar population formed at $z=5$ and the $A B$ colours of the Sun $(B-K)_{\odot}=0.15,(R-K)_{\odot}=-0.76,(V-K)_{\odot}=-0.33$. These rescaled measurements are shown with empty symbols.

To avoid excessive confusion in the plot, some points do not represent a single cluster but are based on different samples: 32 groups and clusters from Sanderson \& Ponman (2003), 8 groups and clusters from Hradecky et al. (2000), 16 clusters from Carlberg et al. (1997) grouped in 4 redshift bins, 105 clusters from Girardi et al. (2000), 12 clusters from Cirimele et al. (1997), 13 clusters from Lin et al. (2003), 180 clusters from Andernach et al. (2004), and 9 clusters from Rines et al. (2004). In such cases, the weighted average (and corresponding error) of the sample is plotted in Fig. 9.

Some of the $M / L$ ratios plotted in Fig. 9 were derived with masses estimated from kinematics (crossed points) or from strong/weak lensing (circled points). The solid line traces the expected evolution of the $M / L$ ratio, neglecting any evolution in the dark halo mass, in the assumption that the $M / L$ ratio evolves following the luminosity evolution of the cluster galaxies, for a pure luminosity evolution of a simple stellar population formed at $z=5$ (normalized at $M / L=51$ at redshift zero).

\section{The stellar mass function}

At redshift $\simeq 1$, the $K_{\mathrm{s}}$ band (rest-frame $1 \mu \mathrm{m}$ ) luminosity is still a good tracer of the stellar mass. Therefore, we can draw an estimate of the stellar mass function (MF) of cluster galaxies at redshift $z \simeq 1.2$ from the composite $K_{\mathrm{s}}$ band LF. The $K_{\mathrm{s}}$ band light translates into the stellar mass via the stellar $M / L$ ratio of each galaxy's stellar population, which depends on the galaxy star formation history (SFH) and on its age.

In principle, if we knew which galaxies are contributing to the composite LF (and we measured their photometry in a sufficient number of passbands) we could measure the stellar $M / L$ ratio for each of the galaxies via SED fitting, and directly determine the stellar mass function. However, since the composite LF was determined in a statistical fashion, we also have to statistically evaluate the stellar $M / L$ ratios of cluster galaxies along the LF. To this aim, we used the Cl1252 photometric members selected by T04 to statistically estimate, in each magnitude bin of the LF, the median and scatter (16th-84th percentiles) colours $i_{775}-K_{\mathrm{s}}, z_{850}-K_{\mathrm{s}}$, and $J_{\mathrm{s}}-K_{\mathrm{s}}$. In the assumption that the contributions to the LF from different galaxy populations in $\mathrm{Cl} 0910$ and $\mathrm{Cl} 0848$ are approximately similar to those in Cl1252, these colours obtained along the Cl1252 LF will be representative of those along the composite LF.

We then built a set of 160 synthetic SEDs with the Bruzual \& Charlot (2003) code, with (delayed) exponentially declining SFH with $0.005<\tau<1.5 \mathrm{Gyr}$ and $0.3<$ age $<5$ Gyr, solar metallicity, Salpeter IMF and no reddening. For each magnitude bin, we selected all the models with the appropriate colour (median \pm scatter as above) for that bin, thus determining a rough estimate of the $M / L$ ratios in that magnitude interval. For each of the three colours $i_{775}-K_{\mathrm{s}}, z_{850}-K_{\mathrm{s}}$, and $J_{\mathrm{s}}-K_{\mathrm{s}}$ we extracted 100 realizations of the MF perturbing the number of galaxies in each magnitude bin with a $\sigma$ equal to its Poissonian error and then spreading in mass this perturbed number of galaxies within the above defined $M / L$ ratio range for that magnitude bin. We then considered the median and minimum/maximum MFs over the 100 realizations, for each of the three colours, which give three estimates of the MF which are perfectly consistent.

We thus averaged these three estimates obtaining the MF plotted in Fig. 10; the shaded region corresponds to the average minimum/maximum MFs computed according to the above description. We also show for comparison the MFs for field galaxies at similar redshift (Drory et al. 2004; Fontana et al. 2004), for field galaxies at redshift zero (Cole et al. 2001), and for local cluster galaxies (Balogh et al. 2001) ${ }^{1}$, all arbitrarily rescaled so to match at $M_{\text {stars }} \simeq 5 \times 10^{10} M_{\odot}$. The MF is shown for masses greater than $\simeq 10^{10} M_{\odot}$, which is the

1 The MF from Balogh et al. (2001) was calculated with a Kennicutt IMF, so we applied a correction $\log M_{\text {stars }}^{\text {salper }}=\log M_{\text {stars }}^{\text {kennicutt }}+0.35$. 


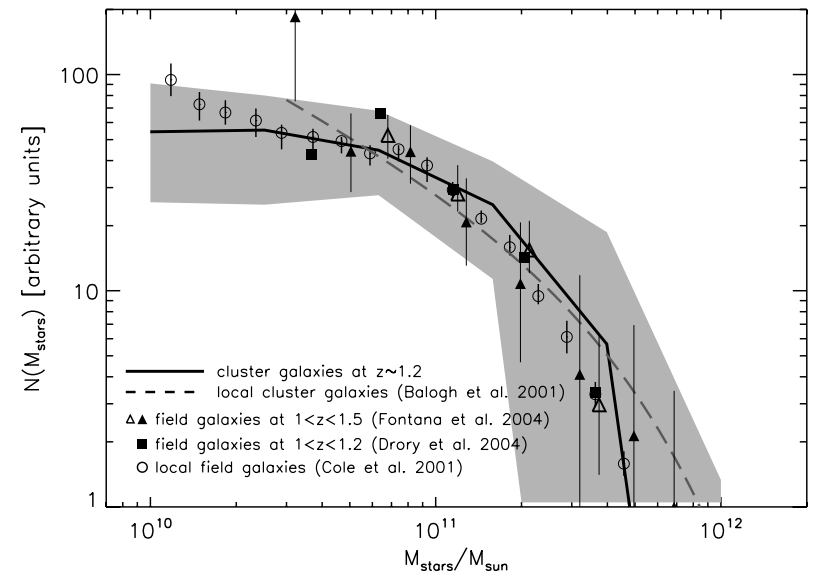

Fig. 10. The stellar mass function as determined from the composite $K_{\mathrm{s}}$ band LF. The shaded area accounts for errors in the $K_{\mathrm{s}}$ band LF and in the stellar mass to $K_{\mathrm{s}}$ light ratio (see text for details). Other determinations of the stellar mass function are shown for reference, as indicated in the legend.

estimated mass completeness determined for our completeness magnitude, considering the $M / L$ ratio of a stellar population formed at $z \simeq 10$ with subsolar metallicity and no dust reddening. The stellar mass corresponding to the characteristic magnitude $K_{\mathrm{s}}^{*}=20.5$ is approximately $10^{11} M_{\odot}$.

Within the uncertainties affecting this MF determination, the shape of the MF of massive objects at redshift $\simeq 1.2$ is not significantly different from the local one as measured from the $2 \mathrm{dF}$ galaxies (Cole et al. 2001). This is in agreement with the very mild evolution of the mass function observed in the redshift range [0:1] both in the field (e.g. Fontana et al. 2004) and in clusters (e.g. Kodama \& Bower 2003). The presence of massive objects $\left(M_{\text {stars }} \geq 10^{11} M_{\odot}\right)$ is independently confirmed at least in Cl1252 from SED fitting on 9 passbands from restframe NUV to NIR (Rosati et al., Rettura et al., in preparation). However, we note that by probing galaxy clusters we are only sensitive to the evolution of the shape of the MF, and not to its normalization, whereas field galaxy surveys find the evolution of the MF to become significant at $z \geq 1$ (Fontana et al. 2004; Drory et al. 2004).

\section{Conclusions}

We have studied the near infrared luminosity function of high redshift cluster galaxies in three X-ray luminous clusters that are among the most distant discovered so far $(1.1<z<1.3)$.

These clusters bear the strongest leverage on evolutionary studies as they probe a redshift regime when the Universe was less than half of its present age. By measuring the $K$ band luminosity function (and galaxy stellar mass function) in these systems, and by comparing it with that at $z \simeq 0$, we can set valuable constraints on the galaxy evolution in dense environments in the redshift range $[0 \div 1]$.

We should note, however, that derived quantities still suffer from large uncertainties, due to small-number statistics and possibly biases in the galaxy populations (due to probing only the cluster cores).

The LF resulting from this work is consistent with previous determinations at similar or lower redshifts, and consolidates a scenario where the evolution of the characteristic magnitude $M^{*}\left(\Delta M^{*} \simeq-1.3\right)$ is consistent with predictions of passive evolution for a stellar population formed at $z>2$. This brightening of $\simeq 1.3 \mathrm{mag}$ is also consistent with the evolution of the mass-to-light ratio $M / L_{K}$ of early-type galaxies, which from fundamental plane studies is found to be $\Delta\left(\ln \left(M / L_{K}\right)\right)=$ $(-1.18 \pm 0.10) z$ (e.g. van der Wel et al. 2006). Moreover, we find that the overall shape of the high redshift LF matches the one of the local cluster galaxies LF, once such a brightening is taken into account.

Similarly, the evolution of the $K$ band LF of field galaxies has been found to be consistent with passive evolution up to $z=1$, with a density evolution lower than $30 \%$ and a brightening of $\Delta M^{*} \simeq 0.5 \div 0.7 \mathrm{mag}$ (e.g. Pozzetti et al. 2003; Drory et al. 2003; however, see also Dahlen et al. 2005).

A direct comparison of the field and cluster LFs at redshift zero, has revealed a significant difference both in the $B$ and in the $K$ band (e.g. Balogh et al. 2001; De Propris et al. 2003) amounting to $\simeq 0.3 \mathrm{mag}$ in $M^{*}$. While there is a hint of exceeding very bright galaxies in our distant clusters with respect to the field, our error bars do not allow us to make such a claim.

For C11252, for which the best quality data and extended wavelength coverage are available, we attempted a separation of the contributions of late and early type galaxies to the bright end of the LF. We find that, already at $z \simeq 1.2$, the bright end of the LF appears to be dominated by early type galaxies selected either on the basis of their morphological appearence or of their spectrophotometric properties.

Using the individual LFs for the three clusters, we calculated the $K$ band cluster $M / L$ ratios making use of X-ray mass profiles derived in Ettori et al. (2004). The $M / L$ ratio tends to be smaller than the typical value at redshift zero, as expected on the basis of pure luminosity evolution of the cluster galaxies stellar populations. However, a much larger sample would be needed for a detailed investigation of the evolution of the cluster $M / L$ ratio.

Finally, from the composite $K_{\mathrm{s}}$ band LF we have estimated the stellar mass function of cluster galaxies. The observed $K_{\mathrm{s}}$ band light at $z \simeq 1.2$ corresponds approximately to the restframe $z$ band light, and is considered as a good tracer of the stellar mass. We have outlined in the introduction how the determination of the stellar mass function at high redshift sets strong constraints on the evolution of massive galaxies. While the early formation epoch of the bulk of the stars in massive galaxies is now generally established by several observations, the epoch of the major mass assembly can only be assessed by studying the redshift evolution of the mass function. Our study shows that the massive $\left(M_{\text {stars }}>10^{10} M_{\odot}\right)$ galaxy populations in massive high redshift clusters have not significantly changed since $z \simeq 1$, apart from passive evolution of their stars, thus extending previous results at lower redshifts. The shape of the stellar mass function at $z \simeq 1.2$ is found to be consistent with the one observed in local clusters, within our uncertainties. 
The high-mass end of the LF, made of giant $\left(M_{\text {stars }}>\right.$ $\left.10^{11} M_{\odot}\right) \mathrm{E} / \mathrm{S} 0$ galaxies, is already in place at $z \simeq 1.2$. This points toward an early assembly of the galaxy mass, mostly completed before $z \simeq 1$, thus implying that the bulk of merging activity for massive galaxies in clusters has to occur at much earlier epochs.

This might appear not surprising as field studies have found evidence of a similar early assembly of massive galaxies with most of the stellar mass already assembled in systems more massive than the local characteristic mass by redshift 1 (e.g. Fontana et al. 2004; Conselice 2005). Since galaxy evolution in clusters is expected to be faster than in the field in hierarchical galaxy formation scenarios, an even milder evolution of the mass function of cluster galaxies is expected. The similarity between the shape of the MF we have found at $z \simeq 1.2$ in rich clusters and in the field sets upper limits on the difference of formation time-scales of stellar populations in high and low density environments.

For example, if we use the star formation histories for early type galaxies in different environments, for different stellar masses, as derived from the fossil record data (Thomas et al. 2005), our results would imply an age difference of $\leq 2 \mathrm{Gyr}$.

The situation is probably different for lower mass galaxies, however it remains difficult to probe the mass function significantly lower than $10^{10} M_{\odot}$ at $z>1$.

Among the caveats in our study, we should mention the so-called progenitor bias. Since we have considered the whole cluster galaxy population, our work is in principle not affected by the progenitor bias referred to when dealing with selected populations of galaxies (generally early-types, van Dokkum \& Franx 2001). It remains true that galaxy populations in clusters at high redshift might not be directly comparable to local ones (e.g. Kauffmann \& Charlot 1998a), and that the high-redshift clusters we are observing might not be the progenitors of the local X-ray luminous clusters (Kauffmann 1995), leading to an underestimated evolution.

With the aid of multicolour photometry, including Spitzer/IRAC bands, we can now directly estimate the stellar masses of high redshift cluster galaxies, as well as approximate ages of their stellar populations, and push these studies out to $z=1.4$ (Mullis et al. 2005; Stanford et al. 2005), thus probing an epoch which is thought to be crucial for the formation of massive clusters. This work will stimulate significant progress in discriminating between different formation scenarios.

Acknowledgements. We thank T. Kodama for providing us with his elliptical galaxy evolution models, and P. Popesso for sharing her results prior to publication. We also thank the anonymous referee for useful comments which improved the presentation of this work. V.S. thanks G. De Lucia, M. Esposito, M. Pannella, M. Paolillo, and A. Rettura for helpful discussions and comments. V.S. gratefully acknowledges support from the European Social Fund through a Ph.D. grant, and from the ESO Director General Discretionary Fund program. SAS's work was performed under the auspices of the US Department of Energy, National Nuclear Security Administration by the University of California, Lawrence Livermore National Laboratory under contract No. W-7405- Eng-48. S.T. received support from the Danish Natural Science Research Council. This work made use of observations of the
GOODS-S field carried out using the Very Large Telescope at the ESO Paranal Observatory under Program ID: LP168.A-0485.

\section{References}

Adami, C., Mazure, A., Biviano, A., Katgert, P., \& Rhee, G. 1998a, A\&A, 331, 493

Adami, C., Mazure, A., Katgert, P., \& Biviano, A. 1998b, A\&A, 336, 63

Andernach, H., Plionis, M., L'opez-Cruz, O., Tago, E., \& Basilakos, S. 2004 [arXiv: astro-ph/0407098]

Andreon, S. 2001, ApJ, 547, 623

Andreon, S. 2004, A\&A, 416, 865

Andreon, S., \& Pelló, R. 2000, A\&A, 353, 479

Andreon, S., Punzi, G., \& Grado, A. 2005, MNRAS, 360, 727

Bahcall, N. A., \& Comerford, J. M. 2002, ApJ, 565, L5

Bahcall, N. A., Lubin, L. M., \& Dorman, V. 1995, ApJ, 447, L81

Balogh, M. L., Christlein, D., Zabludoff, A. I., \& Zaritsky, D. 2001, ApJ, 557, 117

Balogh, M. L., Navarro, J. F., \& Morris, S. L. 2000, ApJ, 540, 113

Bardeen, J. M., Bond, J. R., Kaiser, N., \& Szalay, A. S. 1986, ApJ, 304, 15

Barger, A. J., Aragon-Salamanca, A., Smail, I., et al. 1998, ApJ, 501, 522

Benítez, N., Broadhurst, T., Rosati, P., et al. 1999, ApJ, 527, 31

Bertin, E., \& Arnouts, S. 1996, A\&AS, 117, 393

Blakeslee, J. P., Franx, M., Postman, M., et al. 2003, ApJ, 596, L143

Blanton, M., Cen, R., Ostriker, J. P., \& Strauss, M. A. 1999, ApJ, 522, 590

Bower, R. G., Benson, A. J., Malbon, R., et al. 2005

[arXiv: astro-ph/0511338]

Bruzual, G., \& Charlot, S. 2003, MNRAS, 344, 1000

Carlberg, R. G., Yee, H. K. C., \& Ellingson, E. 1997, ApJ, 478, 462

Carlberg, R. G., Yee, H. K. C., Ellingson, E., et al. 1996, ApJ, 462, 32

Cash, W. 1979, ApJ, 228, 939

Cimatti, A., Mignoli, M., Daddi, E., et al. 2002, A\&A, 392, 395

Cirimele, G., Nesci, R., \& Trevese, D. 1997, ApJ, 475, 11

Cole, S., Norberg, P., Baugh, C. M., et al. 2001, MNRAS, 326, 255

Coleman, G. D., Wu, C.-C., \& Weedman, D. W. 1980, ApJS, 43, 393

Colless, M. 1989, MNRAS, 237, 799

Conselice, C. J. 2005 [arXiv: astro-ph/0507146]

Cowie, L. L., Songaila, A., Hu, E. M., \& Cohen, J. G. 1996, AJ, 112, 839

Dahlen, T., Mobasher, B., Somerville, R. S., et al. 2005 [arXiv: astro-ph/0505297]

David, L. P., Jones, C., \& Forman, W. 1995, ApJ, 445, 578

Davis, M., Efstathiou, G., Frenk, C. S., \& White, S. D. M. 1985, ApJ, 292, 371

De Lucia, G., Poggianti, B. M., Aragón-Salamanca, A., et al. 2004, ApJ, 610, L77

De Propris, R., Colless, M., Driver, S. P., et al. 2003, MNRAS, 342, 725

De Propris, R., Eisenhardt, P. R., Stanford, S. A., \& Dickinson, M. 1998, ApJ, 503, L45

De Propris, R., Stanford, S. A., Eisenhardt, P. R., Dickinson, M., \& Elston, R. 1999, AJ, 118, 719

Demarco, R., Rosati, P., Lidman, C., et al. 2005, A\&A, 432, 381

Dressler, A. 1978, ApJ, 226, 55

Drory, N., Bender, R., Feulner, G., et al. 2003, ApJ, 595, 698

Drory, N., Bender, R., Feulner, G., et al. 2004, ApJ, 608, 742

Ellis, S. C., \& Jones, L. R. 2004, MNRAS, 348, 165

Ettori, S., Tozzi, P., Borgani, S., \& Rosati, P. 2004, A\&A, 417, 13

Fontana, A., Pozzetti, L., Donnarumma, I., et al. 2004, A\&A, 424, 23 
Franx, M., Labbé, I., Rudnick, G., et al. 2003, ApJ, 587, L79

Franx, M., Moorwood, A., Rix, H.-W., et al. 2000, The Messenger, 99, 20

Garilli, B., Maccagni, D., \& Andreon, S. 1999, A\&A, 342, 408

Gavazzi, R., Mellier, Y., Fort, B., Cuillandre, J.-C., \& Dantel-Fort, M. 2004, A\&A, 422, 407

Gehrels, N. 1986, ApJ, 303, 336

Giavalisco, M., Ferguson, H. C., Koekemoer, A. M., et al. 2004, ApJ, 600, L93

Girardi, M., Borgani, S., Giuricin, G., Mardirossian, F., \& Mezzetti, M. 2000, ApJ, 530, 62

Girardi, M., Manzato, P., Mezzetti, M., Giuricin, G., \& Limboz, F. 2002, ApJ, 569, 720

Glazebrook, K., Abraham, R. G., McCarthy, P. J., et al. 2004, Nature, 430,181

Hoekstra, H., Franx, M., Kuijken, K., et al. 2001, ApJ, 548, L5

Hoekstra, H., Franx, M., Kuijken, K., \& van Dokkum, P. G. 2002, MNRAS, 333, 911

Holden, B. P., Blakeslee, J. P., Postman, M., et al. 2005a, ApJ, 626, 809

Holden, B. P., Stanford, S. A., Eisenhardt, P., \& Dickinson, M. 2004, AJ, 127, 2484

Holden, B. P., Stanford, S. A., Eisenhardt, P., \& Dickinson, M. 2005b [arXiv: astro-ph/0503367]

Holden, B. P., van der Wel, A., Franx, M., et al. 2005c, ApJ, 620, L83

Hradecky, V., Jones, C., Donnelly, R. H., et al. 2000, ApJ, 543, 521

Huang, J.-S., Cowie, L. L., Gardner, J. P., et al. 1997, ApJ, 476, 12

Jørgensen, I., Bergmann, M., Davies, R., et al. 2005, AJ, 129, 1249

Kajisawa, M., Yamada, T., Tanaka, I., et al. 2000, PASJ, 52, 61

Kauffmann, G. 1995, MNRAS, 274, 153

Kauffmann, G., \& Charlot, S. 1998a, MNRAS, 294, 705

Kauffmann, G., \& Charlot, S. 1998b, MNRAS, 297, L23

Kinney, A. L., Calzetti, D., Bohlin, R. C., et al. 1996, ApJ, 467, 38

Kneib, J., Hudelot, P., Ellis, R. S., et al. 2003, ApJ, 598, 804

Kodama, T., \& Arimoto, N. 1997, A\&A, 320, 41

Kodama, T., \& Bower, R. 2003, MNRAS, 346, 1

Kodama, T., Yamada, T., Akiyama, M., et al. 2004, MNRAS, 350, 1005

Labbé, I., Franx, M., Rudnick, G., et al. 2003, AJ, 125, 1107

Lidman, C., Rosati, P., Demarco, R., et al. 2004, A\&A, 416, 829

Lin, Y., Mohr, J. J., \& Stanford, S. A. 2003, ApJ, 591, 749

Lin, Y., Mohr, J. J., \& Stanford, S. A. 2004, ApJ, 610, 745

Marinoni, C., \& Hudson, M. J. 2002, ApJ, 569, 101

Massarotti, M., Busarello, G., La Barbera, F., \& Merluzzi, P. 2003, A\&A, 404, 75

McCarthy, P. J., Le Borgne, D., Crampton, D., et al. 2004, ApJ, 614, L9

Mullis, C. R., Rosati, P., Lamer, G., et al. 2005, ApJ, 623, L85

Mullis, C. R., Vikhlinin, A., Henry, J. P., et al. 2004, ApJ, 607, 175

Nagamine, K., Cen, R., Hernquist, L., Ostriker, J. P., \& Springel, V. 2005, ApJ, 627, 608
Nakata, F., Kajisawa, M., Yamada, T., et al. 2001, PASJ, 53, 1139

Nakata, F., Kodama, T., Shimasaku, K., et al. 2005, MNRAS, 357, 1357

Popesso, P., Biviano, A., Böhringer, H., \& Romaniello, M. 2005 [arXiv: astro-ph/0506201]

Postman, M., Franx, M., Cross, N. J. G., et al. 2005, ApJ, 623, 721

Pozzetti, L., Cimatti, A., Zamorani, G., et al. 2003, A\&A, 402, 837

Reiprich, T. H., \& Böhringer, H. 2002, ApJ, 567, 716

Rines, K., Geller, M. J., Diaferio, A., Kurtz, M. J., \& Jarrett, T. H. 2004, AJ, 128, 1078

Rines, K., Geller, M. J., Kurtz, M. J., et al. 2001, ApJ, 561, L41

Rosati, P. 2004, in Clusters of Galaxies: Probes of Cosmological Structure and Galaxy Evolution, 72

Rosati, P., Borgani, S., \& Norman, C. 2002, ARA\&A, 40, 539

Rosati, P., Stanford, S. A., Eisenhardt, P. R., et al. 1999, AJ, 118, 76

Rosati, P., Tozzi, P., Ettori, S., et al. 2004, AJ, 127, 230

Sanderson, A. J. R., \& Ponman, T. J. 2003, MNRAS, 345, 1241

Saracco, P., Longhetti, M., Giallongo, E., et al. 2004, A\&A, 420, 125

Schaeffer, R., Maurogordato, S., Cappi, A., \& Bernardeau, F. 1993, MNRAS, 263, L21

Schechter, P. 1976, ApJ, 203, 297

Stanford, S. A., Eisenhardt, P. R., \& Dickinson, M. 1998, ApJ, 492, 461

Stanford, S. A., Elston, R., Eisenhardt, P. R., et al. 1997, AJ, 114, 2232

Stanford, S. A., Holden, B., Rosati, P., et al. 2002, AJ, 123, 619

Stanford, S. A., Holden, B., Rosati, P., et al. 2001, ApJ, 552, 504

Tanaka, M., Kodama, T., Arimoto, N., et al. 2005

[arXiv: astro-ph/0506713]

Thomas, D., Maraston, C., Bender, R., \& de Oliveira, C. M. 2005, ApJ, 621, 673

Thompson, R. I., Illingworth, G., Bouwens, R., et al. 2005 [arXiv: astro-ph/0503504]

Toft, S., Mainieri, V., Rosati, P., et al. 2004, A\&A, 422, 29

Toft, S., Soucail, G., \& Hjorth, J. 2003, MNRAS, 344, 337

Tran, K. H., van Dokkum, P., Franx, M., et al. 2005, ApJ, 627, L25

Trentham, N., \& Mobasher, B. 1998, MNRAS, 299, 488

Trentham, N., Sampson, L., \& Banerji, M. 2005, MNRAS, 357, 783

Tully, R. B. 2005, ApJ, 618, 214

van der Wel, A., Franx, M., van Dokkum, P. G., et al. 2006, ApJ, 636, L21

van Dokkum, P. G. 2005 [arXiv: astro-ph/0506661]

van Dokkum, P. G., \& Franx, M. 2001, ApJ, 553, 90

van Dokkum, P. G., Franx, M., Fabricant, D., Kelson, D. D., \& Illingworth, G. D. 1999, ApJ, 520, L95

van Dokkum, P. G., Franx, M., Kelson, D. D., \& Illingworth, G. D. 1998, ApJ, 504, L17

van Dokkum, P. G., \& Stanford, S. A. 2003, ApJ, 585, 78

van Dokkum, P. G., Stanford, S. A., Holden, B. P., et al. 2001, ApJ, 552, L101

Williams, R. E., Baum, S., Bergeron, L. E., et al. 2000, AJ, 120, 2735 